\title{
Spatiotemporal profiles of visual processing with and without primary visual cortex
}

\author{
Andreas A. Ioannides ${ }^{a}, *$, Vahe Poghosyan ${ }^{a}$, Lichan Liu ${ }^{a}$, George A. Saridis a , Marco Tamietto ${ }^{\text {b,c }}$, \\ Marc Op de Beeck ${ }^{\mathrm{d}}$, Xavier De Tiège ${ }^{\mathrm{d}}$, Lawrence Weiskrantz ${ }^{\mathrm{e}}$, Beatrice de Gelder ${ }^{\mathrm{b}, \mathrm{f}, * *}$ \\ a Laboratory for Human Brain Dynamics, AAI Scientific Cultural Services Ltd., Office 501 Galaxias Center, 33 Arch. Makarios III Avenue, Nicosia 1065, Cyprus \\ ${ }^{b}$ Cognitive and Affective Neuroscience Laboratory, Tilburg University, 5000 LE Tilburg, The Netherlands \\ c Department of Psychology, University of Torino, 10123 Torino, Italy \\ ${ }^{\mathrm{d}}$ Magnetoencephalography Unit, Laboratoire de Cartographie fonctionnelle du Cerveau (LFCF), Hôpital Erasme, Université Libre de Bruxelles (U.L.B.), Brussels, Belgium \\ e Department of Experimental Psychology, University of Oxford, Oxford, UK \\ f Athinoula A. Martinos Center for Biomedical Imaging, MGH-HMS, Charlestown, MA, USA
}

\section{A R T I C L E I N F O}

\section{Article history:}

Accepted 27 July 2012

Available online 3 August 2012

\section{Keywords:}

Early visual processing

Blindsight

Processing phases

Source imaging

Magnetoencephalography (MEG)

Magnetic field tomography (MFT)

\begin{abstract}
A B S T R A C T
The spatiotemporal profiles of visual processing are normally distributed in two temporal phases, each lasting about $100 \mathrm{~ms}$. Within each phase, cortical processing begins in V1 and traverses the visual cortical hierarchy. However, the causal role of V1 in starting each of these two phases is unknown. Here we used magnetoencephalography to study the spatiotemporal profiles of visual processing and the causal contribution of V1 in three neurologically intact participants and in a rare patient (GY) with unilateral destruction of $\mathrm{V} 1$, in whom residual visual functions mediated by the extra-geniculostriate pathways have been reported. In healthy subjects, visual processing in the first 200 ms post-stimulus onset proceeded in the two usual phases. Normally perceived stimuli in the left hemifield of GY elicited a spatiotemporal profile in the intact right hemisphere that closely matched that of healthy subjects. However, stimuli presented in the cortically blind hemifield produced no detectable response during the first phase of processing, indicating that the responses in extrastriate visual areas during this phase are determined by the feedforward progression of activity initiated in V1. The first responses occurred during the second processing phase, in the ipsilesional high-level visual areas. The activity then spread forward toward higher-level areas and backward toward lower-level areas. However, in contrast to responses in the intact hemisphere, the back-propagated activity in the early visual cortex did not exhibit the classic retinotopic organization and did not have well-defined response peaks.
\end{abstract}

(c) 2012 Elsevier Inc. All rights reserved.

\section{Introduction}

A substantial portion of conscious vision is mediated by the geniculostriate visual pathway, which relays visual information from the retina to the primary visual cortex (V1) and then to the extrastriate cortices. The cortical processing in this pathway begins in V1 at about 40-50 ms post-stimulus onset and proceeds rapidly through low- and mid-level retinotopic extrastriate areas (V2-V5) (Grill-Spector and Malach, 2004; Lamme and Roelfsema, 2000; Poghosyan and Ioannides, 2007). Within the first $100 \mathrm{~ms}$ after stimulus exposure activity spreads

Abbreviations: MFT, magnetic field tomography; RAC, regional activation curve; SP, signal power; UVM, upper vertical meridian; LVM, lower vertical meridian; LHM, left horizontal meridian; RHM, right horizontal meridian; UR, upper right; LR, lower right; UL, upper left; LL, lower left; MOG, middle occipital gyrus; STS, superior temporal sulcus; MTG, middle temporal gyrus.

* Corresponding author. Fax: + 35722028060.

** Correspondence to: B. de Gelder, Cognitive and Affective Neuroscience Laboratory, Tilburg University, 5000 LE Tilburg, The Netherlands. Fax: +31 134662370.

E-mail addresses: a.ioannides@humanbraindynamics.com (A.A. Ioannides), b.degelder@uvt.nl (B. de Gelder). beyond the retinotopic areas proceeding toward high-level, largely non-retinotopic visual areas (Ioannides and Poghosyan, 2012; Liu et al., 2009; Meeren et al., 2008; Okazaki et al., 2008). The second phase of activity through the same areas occurs in the next $100 \mathrm{~ms}$ interval (Ioannides and Poghosyan, 2012; Meeren et al., 2008; Sugase et al., 1999). Within each phase cortical processing begins in V1 and traverses the visual cortical hierarchy (Ioannides and Poghosyan, 2012).

Substantial visual processing is also mediated by the extrageniculostriate pathways that bypass V1 and project directly to the extrastriate cortex. In fact, patients with cortical blindness following damage to V1 can retain some residual visual capability in the absence of awareness ("blindsight" phenomenon) (Poppel et al., 1973; Weiskrantz, 2009b; Weiskrantz et al., 1974). Hemianopic patient GY, who sustained selective early damage to his left V1 can effectively discriminate stimuli presented in his (right) blind hemifield (Cowey and Stoerig, 2004; de Gelder et al., 1999; Schurger et al., 2006; Tamietto et al., 2009, 2010; Weiskrantz et al., 1995; Zeki and Ffytche, 1998). Neuroimaging studies on patient GY have found that his intact right occipital lobe and the intact portions of his left (lesioned) occipital lobe show conventional retinotopic organization (Baseler et al., 1999). However, when stimuli are presented 
in the blind hemifield, activity is restricted to dorsal and/or ventral extrastriate areas and shows abnormal retinotopic organization (Barbur et al., 1993; Goebel et al., 2001; Sahraie et al., 1997; Tamietto et al., 2010; Zeki and Ffytche, 1998).

Notably, however, no study has identified the precise spatiotemporal sequence of visual cortical responses following presentation of stimulus in the cortically blind hemifield, or has directly contrasted these spatiotemporal profiles with those elicited by the same stimuli in the intact hemifield. Furthermore, it is not yet known when and where the first such cortical response appears, or how it spreads thereafter along the adjacent cortical areas, and what, if any, is the causal contribution of V1 to this activity.

In the present study we used magnetoencephalography (MEG) together with a distributed source model (Ioannides et al., 1990; Taylor et al., 1999) to estimate with millisecond time accuracy the spatiotemporal properties of neural activity elicited by checkerboardpattern stimuli placed in different portions of the visual field. We first documented the key spatiotemporal features of visual stimulus processing in three normal subjects, substantially extending the analysis of data reported earlier (Poghosyan and Ioannides, 2007). We then described the neural processing in the intact and damaged hemisphere of GY in response to the same stimuli and in reference to that of the three control subjects.

\section{Methods}

\section{Subjects}

\section{Patient GY}

GY is a 56-year-old man with right hemianopia and "blindsight" following selective damage to his left V1 suffered at the age of 7, as the result of a traumatic brain injury. GY's visual system has been previously tested in behavioral and psychophysiological experiments, as well as with fMRI and diffusion-tensor imaging methods, see (Baseler et al., 1999; Goebel et al., 2001; Tamietto and de Gelder, 2010; Tamietto et al., 2010). The procedure used to map GY's visual field in the current study is described in Supplementary methods.

The MEG experiment with GY was conducted at the MEG unit of the ULB-Hôpital Erasme, Brussels. The resident ethical committee approved the study, which was performed in accordance with the ethical standards laid down in the 1964 Declaration of Helsinki. GY gave informed consent to participate in the study.

\section{Controls}

Three healthy right-handed male subjects aged $26-28$ years, participated in the MEG experiment. All had normal vision. For each subject, the experiment was repeated on three different days. All experiments with normal subjects were conducted at RIKEN Brain Science Institute in Wako-shi, Japan. RIKEN's ethics committee approved the study, and all the subjects gave informed consent. In an earlier publication (Poghosyan and Ioannides, 2007) we reported the analysis of the averaged data from a subset of the runs from this experiment with emphasis on the reproducibility of the results across different experimental days.

\section{Stimuli and experimental design}

\section{Patient GY}

GY was comfortably seated in a magnetically shielded room (MSR). The stimuli were delivered via a DLP projector (Model PT-D7700E, Panasonic, New Jersey, USA) located outside the MSR. Images were back-projected on a screen inside the MSR via an optical periscope.

Nine different locations of the visual field were stimulated, one at a time, using circular checkerboard patterns. The same parameters were used as for the control subjects for luminance and contrast levels (see Controls section), with slight modifications in size and locations made to ensure that the stimuli presented in the right visual hemifield fell entirely within the blind field of GY. Specifically, each stimulus had a radius of $4^{\circ}$ and a check size of $1^{\circ}$ (Fig. 1A). Normally the stimuli were presented centered on the visual vertical meridian $\left(3.6^{\circ}\right.$ above and below the fixation), horizontal meridian ( $10^{\circ}$ to the left and right of the fixation) and in the visual field quadrants $10^{\circ}$ horizontally and $3.6^{\circ}$ vertically off the visual meridians: on the upper vertical meridian (UVM), lower vertical meridian (LVM), left horizontal meridian (LHM) and right horizontal meridian (RHM), and in the upper right (UR), lower right (LR), upper left (UL) and lower left (LL) visual field quadrants. The central (near-foveal) stimulus was centered $1.5^{\circ}$ to the left of the fixation in order to be entirely within the intact portion of the visual field. The stimulus in the UR quadrant was centered slightly closer $\left(1^{\circ}\right)$ to the horizontal meridian (at $2.6^{\circ}$ above the meridian) than the homologous stimulus in the UL quadrant. In this work we focus on the
A

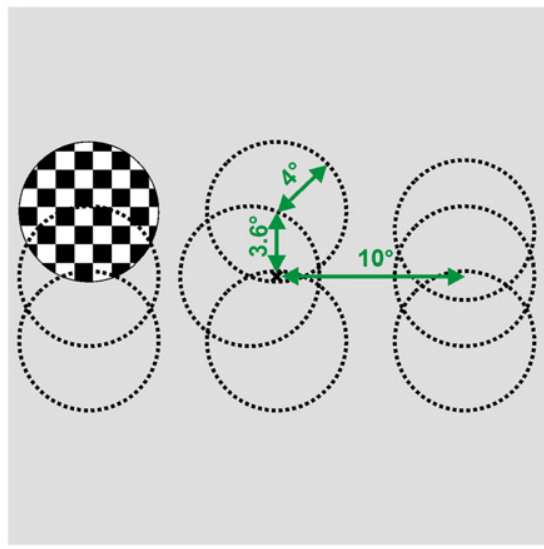

B

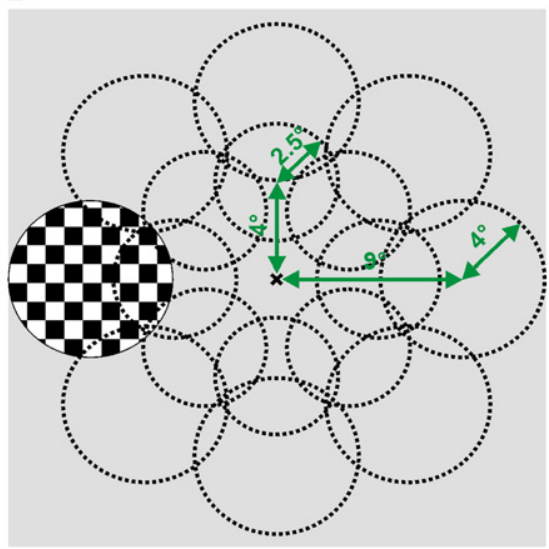

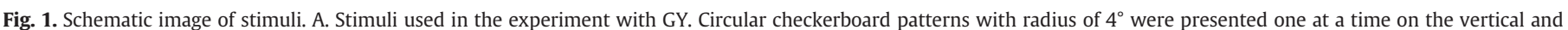

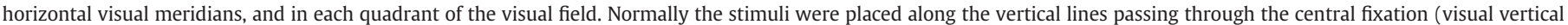

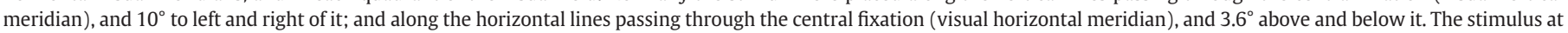

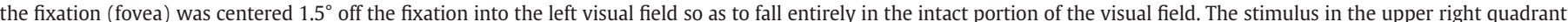

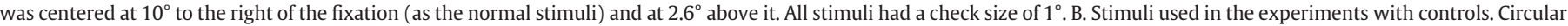

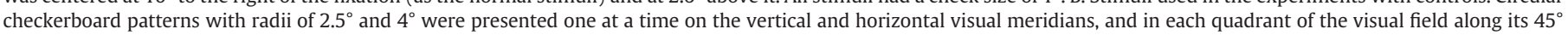
diagonals in random order at $4^{\circ}$ and $9^{\circ}$ eccentricities, respectively. All stimuli had a check size of $1^{\circ}$. 
comparison between the responses to stimuli falling entirely within the blind part of the right visual hemifield and within the homologous locations of the sighted left hemifield. The results from the vertical meridian stimulations are primarily used for delineation of the early visual areas; we will not consider further the data from the near-foveal stimulation in the current manuscript.

In one run, each visual field location was stimulated 10 times. Five such runs were recorded, thus across the runs each visual field location was stimulated 50 times. Locations were stimulated in random order. Each stimulus was displayed for $500 \mathrm{~ms}$, with an interstimulus interval randomized between 400 and $600 \mathrm{~ms}$. The subject was instructed to fixate on a small red cross at the center of the display throughout the run and to try not to make any eye movements. GY was questioned at the end of each of the 5 runs about his visual experience. In all occasions GY reported no awareness whatsoever of visual stimuli presented in his blind hemifield.

\section{Controls}

The subjects were comfortably seated in a MSR. The stimuli were delivered via a fiber-optic goggle system (Avotec Inc., Jensen Beach, FL, USA), which subtended $30^{\circ} \times 23^{\circ}$ of visual angle.

Sixteen different locations of the visual field were stimulated, one at a time, using circular checkerboard patterns, with the check size of $1^{\circ}$ (Fig. 1B). The luminance of the dark and light checks, as measured with a photometer, was 5 and $90 \mathrm{~cd} / \mathrm{m}^{2}$ respectively, thus the Michelson contrast and mean luminance of the checkerboard pattern stimuli were $89 \%$ and $47.5 \mathrm{~cd} / \mathrm{m}^{2}$ respectively. Stimuli at two different eccentricities, $4^{\circ}$ (parafoveal) and $9^{\circ}$ (peripheral), were presented centered on the visual vertical meridian (upper and lower to the fixation), horizontal meridian (left and right to the fixation) and in the visual field quadrants along the $45^{\circ}$ diagonals. Parafoveal and peripheral stimuli had radii of $2.5^{\circ}$ and $4^{\circ}$ respectively. The larger size of the stimulus presented at the peripheral locations did not compensate fully for the cortical magnification factor (Rovamo and Virsu, 1979).

Stimuli at different eccentricities (i.e. at $4^{\circ}$ and $9^{\circ}$ ), and on and off (i.e. in the quadrants) visual meridians were presented in different runs. Hence in each run the stimulus was presented at four visual field locations, a total of 240 times - 60 to each location. Locations were stimulated in random order. Each stimulus was displayed for $500 \mathrm{~ms}$, with an interstimulus interval randomized between 400 and $600 \mathrm{~ms}$. The subject was instructed to fixate on a small red cross at the center of the display throughout the run and to try not to make any eye movements.

\section{MRI data preprocessing and coregistration}

\section{Patient GY}

A high-resolution anatomical MRI of GY was taken with a 3-T Siemens Magneton Allegra scanner in Erlangen, Germany. Cortical reconstruction and volumetric segmentation were performed using a fully automated analysis pipeline provided with the Freesurfer image analysis suite, which is documented and freely available for download online (http:// surfer.nmr.mgh.harvard.edu/). GY's lesion was manually traced on the high-resolution MRI and was projected on to the cortical surface using a software utility developed in-house (see Supplementary methods).

The positions of the MEG sensors were co-registered to the subject's anatomical MRI using the following procedure. Before each experiment four head localization coils were attached to the scalp of GY. The entire surface of his head, together with the head localization coils, was digitized with respect to anatomical fiducials (nasion, right and left tragus) using a 3D digitizer (Polhemus 3Space/ Fastrak, USA). All the digitized points were then fit to the MRI head shape, providing the position of the MEG sensors with respect to the subject's MRI with an estimated accuracy of $3 \mathrm{~mm}$.

\section{Controls}

The high-resolution anatomical MRIs of control subjects were taken with a 1.5-T Siemens Magneton Symphony scanner in Tokyo, Japan. The cortical reconstruction and volumetric segmentation were performed using the same fully-automated Freesurfer analysis pipeline as was used for GY.

The co-registration of MEG and MRI data for each control subject was performed using a special method that provides a co-registration accuracy of about $1 \mathrm{~mm}$ (Hironaga and Ioannides, 2002). The details of the performed procedures can be found elsewhere (Poghosyan and Ioannides, 2007).

\section{Supporting fMRI experiment}

The supporting fMRI experiment with control subjects was conducted using a Varian Unity Inova 4-T whole-body MRI system (Varian NMR Instruments, Palo Alto, CA) equipped with a Magnex head gradient system (up to 3.4 gauss/cm with a rise time of $\sim 160 \mu$ s, Magnex Scientific Ltd., Abingdon, UK). During the recording, the subject's head was fixed using a bite bar. Two pressure sensors were also placed around the subject's head to monitor any head motion. The functional data were acquired using multishot $\mathrm{T} 2 *$-weighted echo-planar imaging pulse sequence $\left(\mathrm{TR}=600 \mathrm{~ms} ; \mathrm{TE}=15 \mathrm{~ms}\right.$; flip angle $=40^{\circ}$, sampling bandwidth $=$ $102.4 \mathrm{kHz}$ ). More details of the fMRI experiment can be found elsewhere (Cheng et al., 2001).

The stimuli were delivered to the subject's eyes via a pair of independently controlled fiber-optic bundles (Silent Vision binocular glasses, Avotec Inc., Jensen Beach, FL, USA) subtending $30^{\circ} \times 23^{\circ}$ of visual angle. To identify the borders between V1 and V2 visual areas vertical meridian was stimulated using a vertically oriented bow tie-shaped black/white checkerboard with contrast reversal at $7.5 \mathrm{~Hz}$ on a gray background.

\section{MEG data acquisition and preprocessing}

\section{Patient GY}

MEG signals for GY were collected at the MEG unit of the ULB-Hôpital Erasme, Brussels using the 306-channel (204 planar gradiometers and 102 magnetometers) whole-head Vectorview MEG system (Elekta Neuromag Oy, Helsinki, Finland). The detailed characteristics of the system can be found elsewhere (De Tiège et al., 2008). The MEG signals were recorded with a passband of $1-330 \mathrm{~Hz}$ at a sampling rate of $1000 \mathrm{~Hz}$. Vertical and horizontal electrooculogram (EOG), and electrocardiogram (ECG) were recorded in synchrony with MEG signals. The position of the subject's head relative to MEG sensors was continuously monitored by feeding current to four head localization coils attached to the scalp.

\section{Controls}

MEG signals for normal subjects were collected at the Laboratory for Human Brain Dynamics (1998-2009) at RIKEN Brain Science Institute, Japan using a 151 gradiometer whole-head Omega system (CTF/ VSM MedTech Ltd., BC, Canada). In synchrony with the MEG signals, vertical and horizontal EOG and ECG were recorded at the same sampling rate of $1250 \mathrm{~Hz}$. In addition, eye position during the whole run was recorded with an eye tracking system (Avotec Inc., Jensen Beach, FL, USA) mounted on the goggles.

The subjects' head location was recorded at the beginning and end of each run, which lasted about 4 min. Average head movement of each subject during a run was around $1-2 \mathrm{~mm}$. Runs in which movement exceeded $4 \mathrm{~mm}$ were repeated.

Offline, the MEG signals were converted to a 3rd-order synthetic gradient, low-pass filtered at $200 \mathrm{~Hz}$, and resampled at $625 \mathrm{~Hz}$. Trials where the eyes deviated more than $1^{\circ}$ from the fixation cross, or where the subject blinked, were identified using the eye tracker and EOG data, and were removed from further analysis. 


\section{Data analysis}

All MEG and MRI data (from GY and the three control subjects) were analyzed in the same way at the Laboratory for Human Brain Dynamics at AAI Scientific Cultural Services Ltd., Cyprus. Independent component analysis (Jahn et al., 1999) in conjunction with ECG data was used to remove the cardiac artifacts. The cleaned MEG signals were then divided into $600 \mathrm{~ms}$ trials, from -200 to $400 \mathrm{~ms}$ with respect to stimulus onset.

\section{Tomographic source analysis}

Magnetic field tomography (MFT) (Ioannides et al., 1990; Taylor et al., 1999) was applied to single trial MEG signals to estimate the three-dimensional distribution of current sources throughout the brain, independently for each snapshot (time point of a single trial) of data. MFT uses a non-linear algorithm for solving the biomagnetic inverse problem. It estimates the primary current distribution $\vec{j}(\vec{r})$ inside the source space $S$ with the following recursive expression:

$\vec{j}_{n+1}(\vec{r})=\left|j_{n}(\vec{r})\right| \sum_{k=1}^{N} A_{k}^{n} \vec{\phi}_{k}(\vec{r}) w_{0}(\vec{r}), \quad \vec{r} \in S$

where $A_{k}^{n}$ are scalar expansion coefficients, which must be determined together with $\vec{j}_{n}(\vec{r})$ in every iteration, $w_{0}(\vec{r})$ is the initial a priori probability weight function, which in practice is determined independently for each experimental arrangement (i.e. for each run and subject) through a training session with a known set of current dipoles spread throughout the source space, and $\vec{\phi}_{k}(\vec{r})$ are the lead field functions, which relate $\vec{j}(\vec{r})$ to the magnetic field $b_{k}$ measured at $k^{\text {th }}$ MEG sensor:

$b_{k}=\int_{S} \vec{\phi}_{k}(\vec{r}) \bullet \vec{j}(\vec{r}) d \vec{r}, k=(1, \ldots, N)$

By combining Eqs. (1) and (2) in every iteration we obtain a set of equations:

$b_{i}=\sum_{k=1}^{N} A_{k}^{n} \int_{S}\left(\vec{\phi}_{i}(\vec{r}) \bullet \vec{\phi}_{k}(\vec{r})\right) w_{0}(\vec{r})\left|j_{n}(\vec{r})\right| d \vec{r}, i=(1, \ldots, N)$

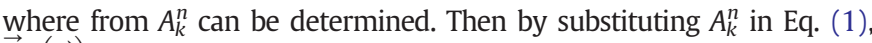
$\vec{j}_{n}(\vec{r})$ is found. To overcome the ill-posedness of the Eq. (3) in the software implementation of MFT Tikhonov regularization is used, with the regularization parameter pre-determined using computer simulations. In the current study and in all our earlier studies the current source distribution, $\vec{j}(\vec{r})$, has been estimated using one iteration $\left(\vec{j}_{1}(\vec{r})\right)$, with a starting choice of $\left|\vec{j}_{0}(\vec{r})\right|=1$. The detailed analysis of lead field expansions has shown that the standard form of MFT has optimal stability and sensitivity for localized distributed sources (Taylor et al., 1999). In contrast to linear inverse methods, standard MFT relies less on the raw lead fields and makes more use of the measured data. Specifically, it makes less assumptions than the linear methods, because in the standard MFT only the direction of the current source distribution is expressed by a linear expansion in lead fields, while the linear methods presuppose that both direction and strength of the current source distributions can be expressed in a single linear expansion in the lead fields.

For each subject, five partially overlapping source spaces, covering the left and right hemispheres, and front, back and top of the brain have been defined. MFT was used to estimate the current source distribution separately in each source space using signals from the 90 MEG sensors best covering the source space under examination. The MFT estimates from all five source spaces were then combined together, with a weighting that reflects the sensitivity profile of the inverse solution at each source space point, yielding a final source space that covers the entire brain.

\section{Post-tomographic source analysis}

Sets of single trial MFT estimates were then used to compute a time-varying signal-to-noise ratio (SNR) (Laskaris and Ioannides, 2001; Raz et al., 1988) map for each stimulated location of the visual field, experimental day and subject. The SNR was computed using the following formulae:

$$
\begin{aligned}
& \bar{X}=\frac{\sum_{i=1}^{N} X_{i}(t, p)}{N}, \frac{N P=\sum_{i=1}^{N}\left\|\bar{X}-X_{i}(t, p)\right\|_{L 2}^{2}, S P}{p(N-1)}=\frac{1}{p}\|\bar{X}\|_{L 2}^{2}-\frac{1}{N} N P, \\
& S N R=\frac{S P}{N P}
\end{aligned}
$$

where $X_{i}(t, p)$ is a $p$ ms interval centered at $t$ ms latency and extracted from the $i^{\text {th }}$ single trial. The SNR values for each brain voxel were computed using $20 \mathrm{~ms}$ windows (i.e. $p=20$ ) moving from -190 to $390 \mathrm{~ms}$ (i.e. $t=-190, \ldots, 390$ ). Each SNR map was generated from a set of 50 to 60 single trial MFT estimates and in all figures presented in this paper SNR values above 0.2 are shown. This SNR threshold was selected so as to eliminate all pre-stimulus activity in control subjects. The methodology was successfully applied in a number of recent studies, always showing peak activations in V1 in close agreement with the known retinotopic organization of V1: Stimuli falling in one visual hemifield (i.e. UR, RHM, LR, LL, LHM and UL) elicited activations in the contralateral brain hemisphere, while stimulations of the lower and upper visual field locations (i.e. UVM, UR, LR, LVM, LL and UL) activated regions dorsal and ventral to calcarine sulcus respectively.

\section{Regions of interest and regional time courses}

SNR maps were visually inspected timeslice-by-timeslice and brain regions that exhibited distinct activations consistently across the three experimental days were identified. For each subject the centroids of such activations were designated as centers of spherical regions of interest (ROI) with a radius of $1 \mathrm{~cm}$. The principle direction for each ROI, which is the dominant orientation of the current density vectors in that ROI was calculated using circular statistics (Fisher, 1993), an established framework for identification of statistically significant distributions based on both magnitude and direction of vectors (Ioannides et al., 2005; Poghosyan and Ioannides, 2007, 2008). For control subjects and the unaffected visual hemifield of the patient GY, ROIs were sought starting from the first suprathreshold activation on each SNR map till $100 \mathrm{~ms}$ after stimulus onset. For three stimuli (UR, RHM and LR) that fell in the cortically blind hemifield of GY, ROIs were defined from the first suprathreshold responses elicited by them; for each of these three stimuli the first response was identified at latencies longer than $130 \mathrm{~ms}$ and within the high-level visual cortex. Three such ROIs were defined in the ipsilesional left hemisphere. Three additional ROIs were defined, one for each "unseen" stimulus, in the same (ipsilesional left) hemisphere from the first responses in the early visual cortex (area V2 or V3). Next we identified three ROIs in each control subject corresponding to the three high-level ipsilesional ROIs of GY. Such ROIs were defined based on anatomical and functional criteria. Anatomically they had to be within $1 \mathrm{~cm}$ from their respective patient ROIs in the Talairach space (Talairach and Tournoux, 1988) and have similar localization with respect to sulcal/ gyral landmarks - the ROIs were around middle occipital gyrus (MOG), superior temporal sulcus (STS) and middle temporal gyrus (MTG). Functionally, their localization and latencies had to be reproducible across the three experimental days.

Regional activation curves (RAC) were then generated for all V1 ROIs in intact hemispheres and the three high-level ipsilesional ROIs, for both controls and the patient. RAC describes the activation time course of an ROI along its principal direction and were generated from single trial MFT estimates by integrating, for each time point of $1.6 \mathrm{~ms}$, the projections of the current density vectors along the principle direction in the ROI. To quantify the signal content in an ensemble of single trial RACs we computed their signal power (SP) (Laskaris and Ioannides, 2001; Raz et al., 1988) using a 10 ms moving window. SP time courses were 
generated separately for each ROI, stimulated visual field location and subject.

\section{Computer simulations}

The data for GY and control subjects were recorded at different sites using two different MEG systems. Our analysis methodology is robust enough to adequately deal with this limitation, as earlier studies have demonstrated (Ioannides et al., 2002). We nevertheless confirm here, using computer simulations, the consistency of the source localization across the two systems and the ability to identify and distinguish two concurrently active nearby sources (in V1 and V2, $0.9 \mathrm{~cm}$ apart). The details of computer simulations are provided in the Supplementary methods.

\section{Results}

\section{Analysis of simulated data}

The analysis of simulated data showed that the localizations obtained from GY and our controls subjects can be directly compared despite the differences in the MEG hardware used to record the signals in each case, and that the employed methodology is capable of identifying concurrently active source in V1 and V2 (see Supplementary results and Supplementary Figs. 1 and 2).

\section{Source analysis in the intact hemispheres}

\section{Controls}

The first SNR activation (above the threshold of 0.2 ) in both brain hemispheres of the normal subjects was identified between 40 and $70 \mathrm{~ms}$ post-stimulus, with its peak localized in V1. These peak activations were in good agreement with the known retinotopic organization of V1 (Holmes, 1945; Horton and Hoyt, 1991): the activations were contralateral to stimulus hemifield, stimuli in the lower and upper visual fields activated regions dorsal and ventral to calcarine sulcus respectively, and activations in response to parafoveal stimulations were localized more posterior and closer to the occipital pole compared to activations elicited by peripheral stimulations, which were deeper in the calcarine. The responses elicited by stimuli presented along the visual meridians (i.e. UVM, RHM, LVM and LHM) were localized within 2-3 mm of the corresponding fMRI representations, which were obtained for normal subjects in an independent fMRI experiment (Fig. 2A).

Following this initial response in V1 the activity rapidly spread through the early visual cortex, ventrally toward occipitotemporal and dorsally toward posterior parietal brain regions. The results for one normal subject are shown in Fig. 3A (LL stimulation) and 3B (UL stimulation). The points with $50 \%$ or higher probability for cytoarchitectonic areas V4 and V5 are also displayed on the inflated brains. The figure shows that the activity reached the end-level of the classic retinotopic visual cortex, area V4, at $\sim 80 \mathrm{~ms}$ post-stimulus. In agreement with earlier studies (Fylan et al., 1997; Portin et al., 1999; Tzelepi et al., 2001) activity in the early visual cortex was stronger in response to lower than upper visual field stimulations.

\section{Patient GY (intact hemisphere)}

The spatial and temporal pattern of visual responses in the intact hemisphere of GY was similar to that of control subjects (using the same SNR threshold of 0.2). Specifically, the first brain responses to left visual field stimulations were identified in the right V1 at "retinotopically correct" locations between 50 and 70 ms post-stimulus (Fig. 2A right part). Thereafter, the activity rapidly traversed the visual cortical hierarchy (Figs. 3C and D). Again responses were stronger for lower rather than upper visual field stimuli.

\section{Regions of interest in the intact hemispheres}

We used the first $100 \mathrm{~ms}$ of the post-stimulus period to discern notable SNR activation peaks in normal subjects and in the intact hemisphere of GY. For each stimulated location we then defined ROIs centered at the corresponding peaks. In both brain hemispheres of normal subjects, the distribution of the ROIs on the flattened occipital cortex followed the classic visual field maps (Fig. 2A). In normal subjects stimuli were presented in the quadrants and on the meridians of the visual field at two different eccentricities. The borders between early visual areas could thus be estimated by connecting the representations of stimuli placed at different eccentricities on each meridian on the flattened occipital cortex using the following procedure. The ROIs obtained from the first activations elicited by stimuli presented along the upper and lower vertical meridians (UVM and LVM) defined respectively the ventral and dorsal borders between areas V1 and V2. The ROIs obtained from the next activations elicited by the same stimuli (UVM and LVM) defined the borders of V3 and V4. For the data analyzed here the V1V2 border was identified in all hemispheres but the V3-V4 border only in some hemispheres. The ROIs obtained from the first activations elicited by stimuli presented along the left and right horizontal meridians (LHM and RHM) separated the ventral and dorsal portions of V1. The ROIs obtained from the next activations elicited by the same (LHM and RHM) stimuli defined the borders between areas V2 and V3 in the right and left brain hemispheres respectively. This putative border allocation was substantiated by three independent results. First, stimuli presented in visual field quadrants elicited activity in early visual areas in-between the corresponding representations of vertical and horizontal meridians. The entire set of ROIs was perfectly consistent with the retinotopic maps obtained routinely with fMRI. Furthermore, the latencies and the sequence of activations from which the ROIs were defined were in accord with a feedforward spread of activation. Second, as can be seen in Fig. 2A, our V1-V2 border estimates (white lines) are in excellent agreement with the independent fMRI estimates (black lines). Finally, the relative alignment of the retinotopic areas V1V3 defined from our MEG-based source analysis and the areas V4 and V5 defined from the probabilistic cytoarchitectonic maps (Eickhoff et al., 2005) is consistent with the known organization of visual cortex: V4 and V5 fall nicely just outside the retinotopic areas V1-V3.

The range of stimuli used for GY was more limited, but still, in the intact hemisphere the approximate borders between early visual areas can be defined by lines connecting the ROIs for stimuli along the meridians with the occipital pole (right part of Fig. 2B). These ROIs were defined from the early responses at latencies similar to the ones identified in normal subjects. The estimates for the V1-V2 and ventral V2-V3 borders were consistent with the results from the control subjects. Furthermore the first activations elicited by stimuli just off the horizontal meridian fall nicely within the expected cytoarchitectonic area.

\section{Time courses in the intact hemispheres}

To examine the temporal dynamics of individual ROIs for different stimulated locations, we produced RAC-based SP time courses from single trial MFT estimates, for all V1 ROIs and the three "ipsilesional" ROIs, for normal subjects and for the patient (see Regions of interest and regional time courses section). In normal subjects, the V1 time courses for the retinotopically appropriate stimuli were highly reproducible across different experimental days as already reported for quadrant activations (Poghosyan and Ioannides, 2007). Fig. 4 shows V1 time courses for all stimuli; the curves from each of the three experimental days are superimposed demonstrating the reproducibility of responses. The figure shows also the separation into processing phases, defined by the two distinct responses, in the 50-100 and 100-200 ms latency ranges. These results (Fig. 4, see also Fig. 9) together with our recent findings (Ioannides and Poghosyan, 2012) and other converging 

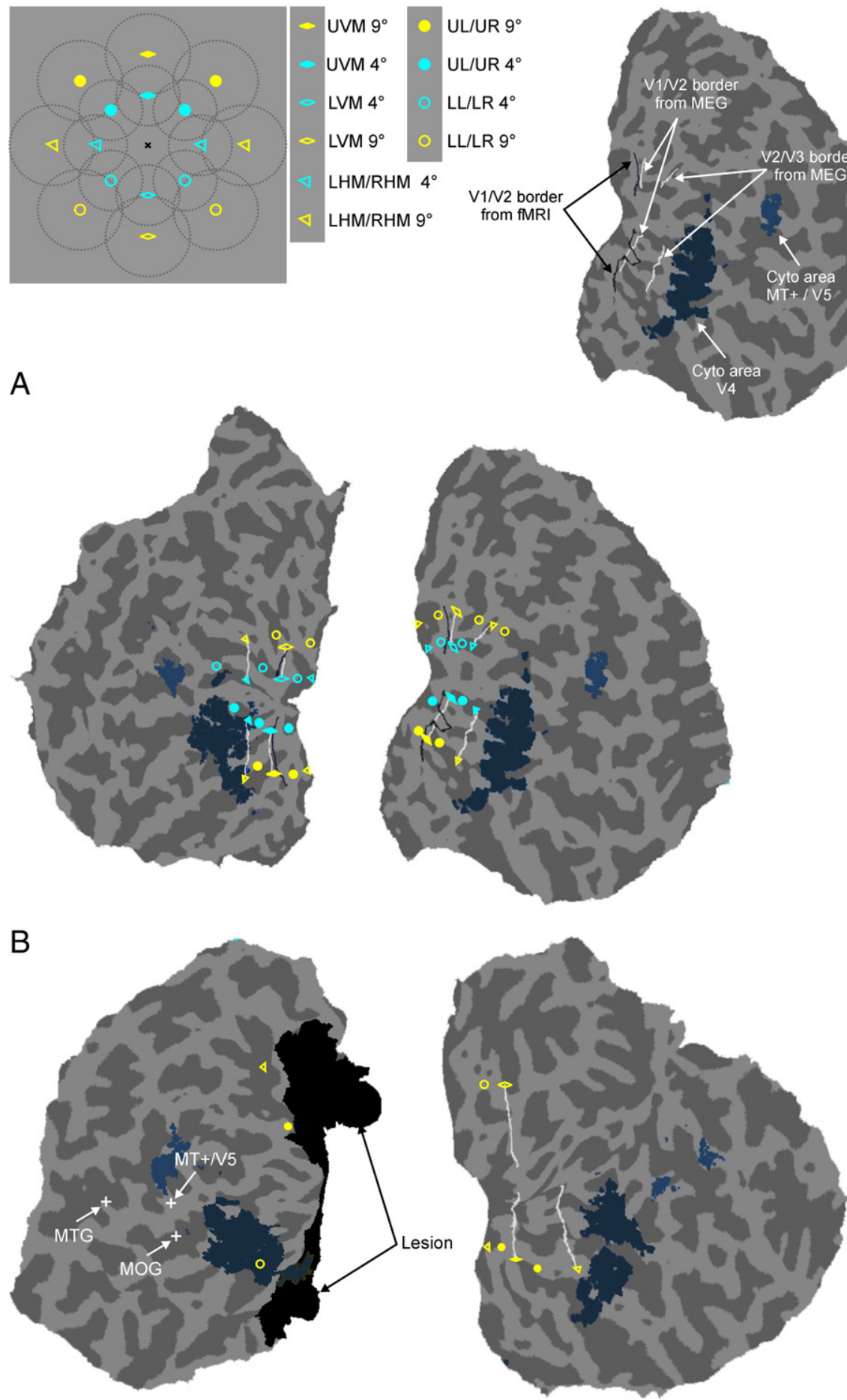

Fig. 2. Distribution of the ROIs on the flattened occipital cortex. The left hemisphere flattened patches are shown on the left and the right hemisphere patches on the right. Colored shapes mark centers of ROIs obtained in response to stimuli presented in different parts of the visual field, as depicted on the upper right part of the figure: Cyan and yellow shapes mark ROIs corresponding to stimuli presented at eccentricities of $4^{\circ}$ and $9^{\circ}$ respectively. Triangles, rhombi and circles indicate ROIs for stimuli presented on the horizontal meridian, vertical meridian and in the quadrants respectively. Filled and empty shapes indicate ROIs for stimuli presented in the upper and lower visual fields respectively. The markings for estimated visual area borders are indicated on the example flattened patch on the upper right part of the figure: White lines indicate borders between early visual areas estimated based on source analysis of MEG data alone. Black lines indicate the borders between areas V1 and V2 estimated in independent fMRI experiments. Putative V4 and V5 areas obtained from the above 50\% cytoarchitectonic probabilistic maps are also indicated on the flattened patches. $\boldsymbol{A}$, Distribution of ROIs in a typical control subject. B. Distribution of ROIs in GY. Crosses mark the locations of the three high-level ROIs in the ipsilesional hemisphere. The black patches show the lesioned portion of the left occipital cortex.

evidence (Liu et al., 2002; Meeren et al., 2008; Sugase et al., 1999) show that in the first $200 \mathrm{~ms}$ after stimulus onset normal visual processing contains at least two phases, spanning roughly the first and second 100-ms post-stimulus intervals respectively. During each phase activity starts in V1 and spreads thereafter in a feedforward direction through the hierarchy of visual cortical areas. In accord with earlier studies 


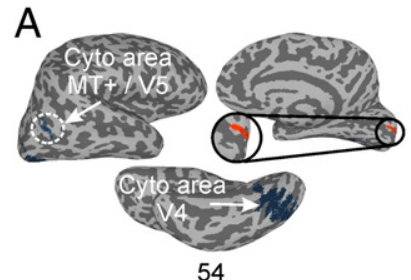

B
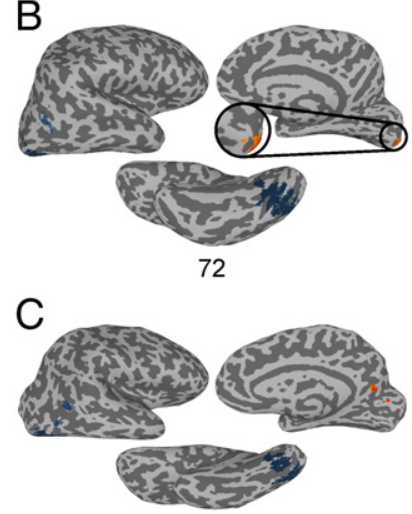

60

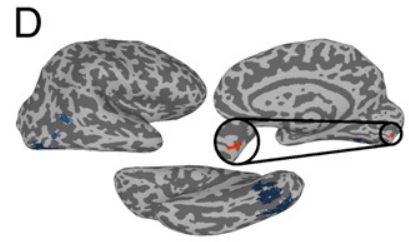

60

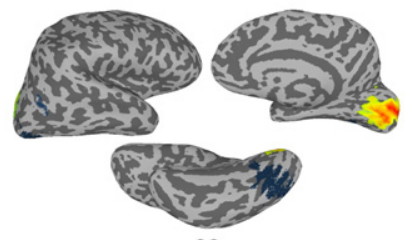

60

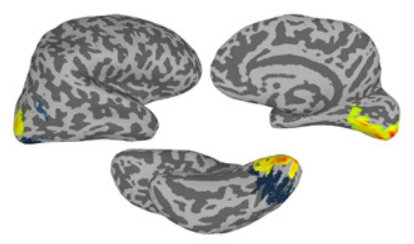

78

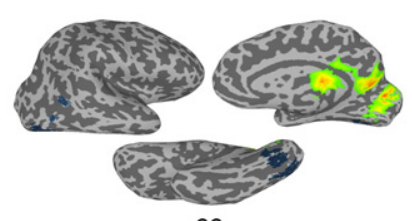

66

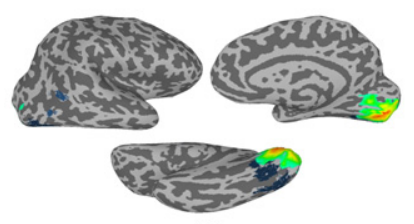

66
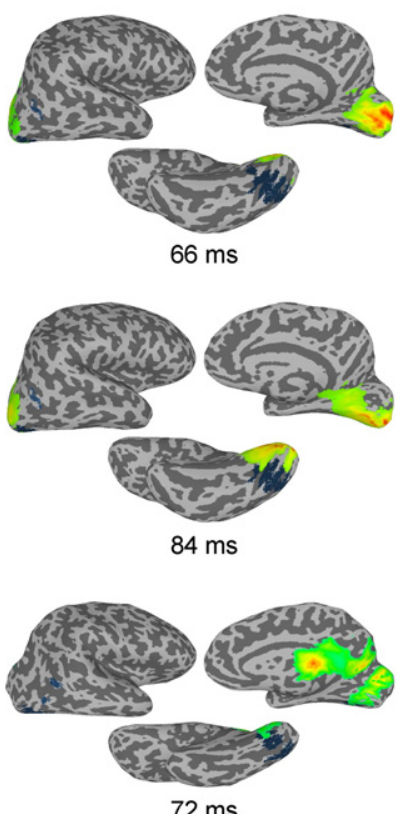

$72 \mathrm{~ms}$

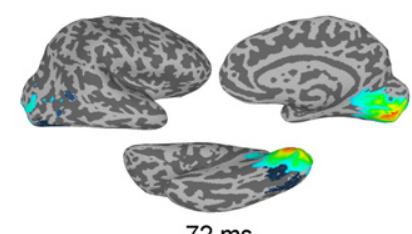

$72 \mathrm{~ms}$

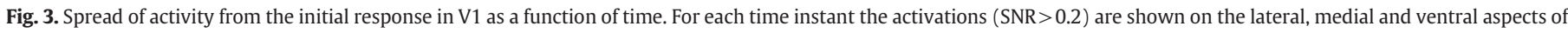

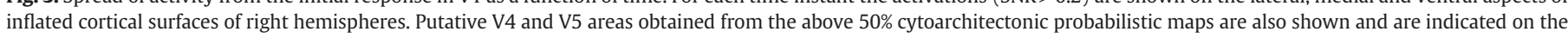

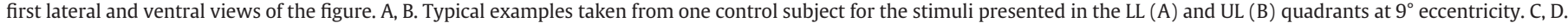
Results taken from GY for the stimuli presented in the LL (C) and UL (D) quadrants.

(Fylan et al., 1997; Portin et al., 1999; Tzelepi et al., 2001) and the evidence from our SNR maps, SP time courses were stronger in response to lower than upper visual field stimuli.

Fig. 5 shows time courses for the patient's intact (right) hemisphere V1 ROIs, defined separately for UVM, UL, LHM, LL and LVM stimuli (see Regions of interest and regional time courses section). No noticeable V1 activation was seen for stimuli presented in the cortically blind right hemifield (not shown). In all V1 ROIs the strongest SNR was found in response to retinotopically corresponding visual field location (shown in Fig. 5). In line with the results from normal subjects, the visually evoked activity in the intact hemisphere contained two distinct responses, at $\sim 70$ and $150 \mathrm{~ms}$ latencies, corresponding very likely to the two-phase visual processing scheme observed in the normal subjects. Furthermore, SP was higher in response to stimuli presented in the lower than upper visual field, especially during the first phase of processing.

\section{Results from the left (ipsilesional) hemisphere of GY}

We studied next the activity elicited by stimuli presented in GY's cortically blind right hemifield (UR, RHM and LR). The most striking difference between the activations in the damaged hemisphere compared to the ones in the intact hemisphere was the absence of activity during the first phase of processing ( $<130 \mathrm{~ms}$ ). Setting the same SNR threshold of 0.2 as the one employed for the control subjects and for the intact hemisphere, the first activations in the damaged hemisphere elicited by stimuli presented in the patient's blind hemifield (right hemifield; UR, RHM and LR) appeared between 140 and $160 \mathrm{~ms}$ post-stimulus in the damaged hemisphere (white crosses in the left part of Figs. 2B, and 6). These first responses in the damaged hemisphere are shown in Fig. 6 superimposed on MRI anatomical slices and inflated cortical surface. For ease of reference the putative MT + V5 according to cytoarchitectonic probability maps (Malikovic et al., 2007) is also displayed on the inflated maps. The three identified activations were localized in the middle occipital gyrus (MOG, upper row in Fig. 6); around the confluence of superior temporal sulcus (STS), middle temporal gyrus (MTG) and MOG (middle row in Fig. 6); and on the border of MTG and STS (lower row in Fig. 6).

The left part of Fig. 2B shows the locations of the peak activity (white crosses) for each of the three areas on the flat map of the damaged hemisphere together with the putative V4 and V5 areas. The absence of retinotopic activations in early visual cortex did not allow us to define the visual area borders in the damaged hemisphere. However, the juxtaposition in Fig. 2B of the left hemisphere with the intact right hemisphere strongly suggests that these first activations in the damage hemisphere are in the high-level visual cortex.

Following these first responses in the damaged hemisphere activity spread forward to occipitotemporal cortex and backward to early visual cortex (Figs. 7A, B and C). The locations of the first responses in the early visual cortex elicited by stimuli presented in the cortically blind right hemifield (UR, RHM and LR) are shown in Fig. 2B (yellow shapes in the left part). The responses to UR and LR stimuli likely fall within area V2 or V3, while the first response to RHM stimuli is within V3A or V7. Although the LR elicited response is in a brain region with above $50 \%$ probability for $\mathrm{V} 4$ based on cytoarchitectonic probabilistic maps, its location relative to calcarine sulcus/lesion and the juxtaposition of left and right hemisphere flat maps indicate that the response is in ventral V2 or V3. However, in contrast to responses in the intact hemisphere (Figs. 7D, E and F) these late 'feedback' activations did not adhere to classic retinotopic organization: responses to upper and lower visual field stimuli were localized in dorsal and 

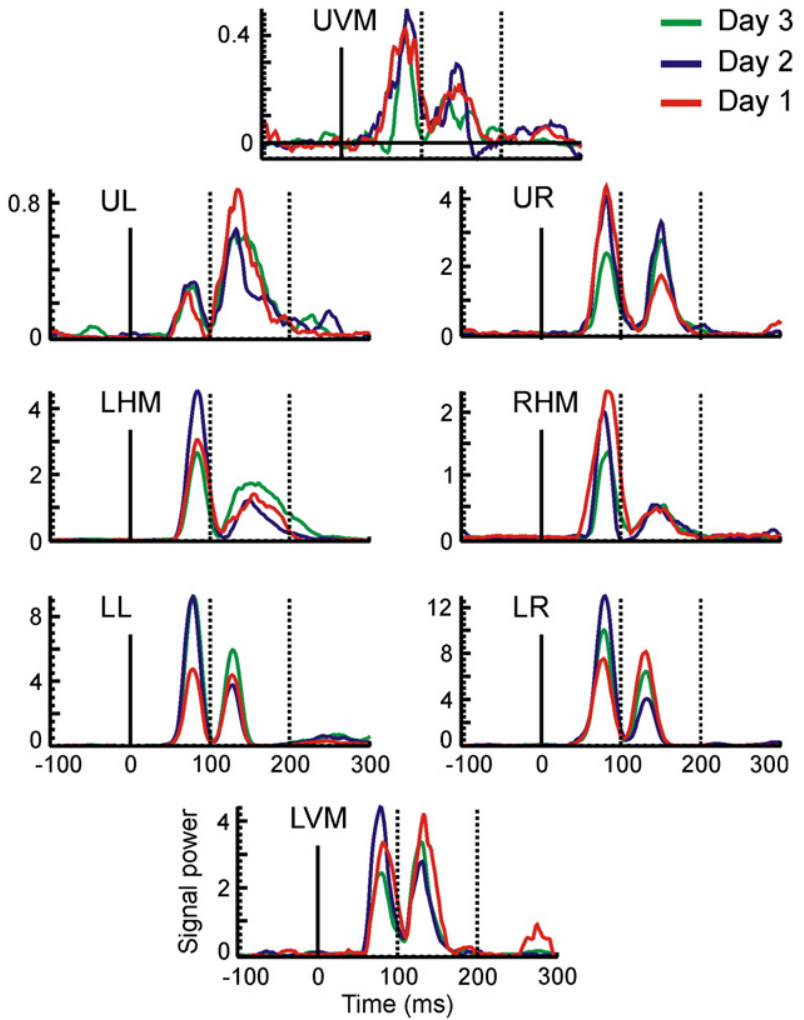

Fig. 4. SP time courses for V1 ROIs in a typical control subject. Time courses obtained from three different experimental days are superimposed (red - day 1, blue - day 2 , green - day 3). Each figure displays time courses for one location of the visual field (printed on its upper left part). The placement of each figure corresponds roughly to the location in the visual field and proceeding in a clock-wise direction - they are UVM, UR, RHM, LR, LVM, LL, LHM and UL. The results for stimuli presented at $9^{\circ}$ eccentricity are shown. Each figure shows the time courses for each day for the ROI corresponding to the stimulated location.

ventral early visual cortex respectively (see the yellow shapes in the left part of Fig. 2B and zoomed insets in Figs. 7A and C). They were also less organized in time (Fig. 8).

Fig. 9 shows SP time courses for the patient in the three high-level ipsilesional ROIs (note the absence of response during the first processing phase, $<130 \mathrm{~ms}$ ). Both left and right visual hemifield stimuli produced prominent responses in these ROIs, confirming that they are located beyond the classic retinotopic cortex and in high-level visual areas. The response latencies of all three ROIs for left (ipsilateral) hemifield stimuli (retinotopically mapped to the intact hemisphere) were very similar, starting around $120 \mathrm{~ms}$ post-stimulus and peaking at $150 \mathrm{~ms}$. The latencies in response to right (contralateral) hemifield stimuli (retinotopically mapped to the damaged hemisphere) were slightly longer, stronger and more variable across stimulus locations.

Finally, we identified three ROIs in each normal subject corresponding to the GY's three ipsilesional high-level ROIs (see Regions of interest and regional time courses section). Fig. 10 shows the SP time courses in these three ROIs in the representative normal subject. The responses in these three left hemisphere ROIs for the normal subjects and GY can be contrasted in the context of two-phase visual processing. The responses in normal subjects (Fig. 10) show two clear visual processing phases: in all three ROIs the responses in the first phase peak around or just before $100 \mathrm{~ms}$ post-stimulus for the contralateral stimuli (right visual field stimuli) and about $20 \mathrm{~ms}$ later for the ipsilateral stimuli. In the second phase, the peak activity is earlier in the left MTG (green lines) at $\sim 150$ ms post-stimulus, while the activity in the other two ROIs peaks at $\sim 200 \mathrm{~ms}$. For the patient no evoked activity was found before $120 \mathrm{~ms}$ in the damaged hemisphere. The latencies of the first responses
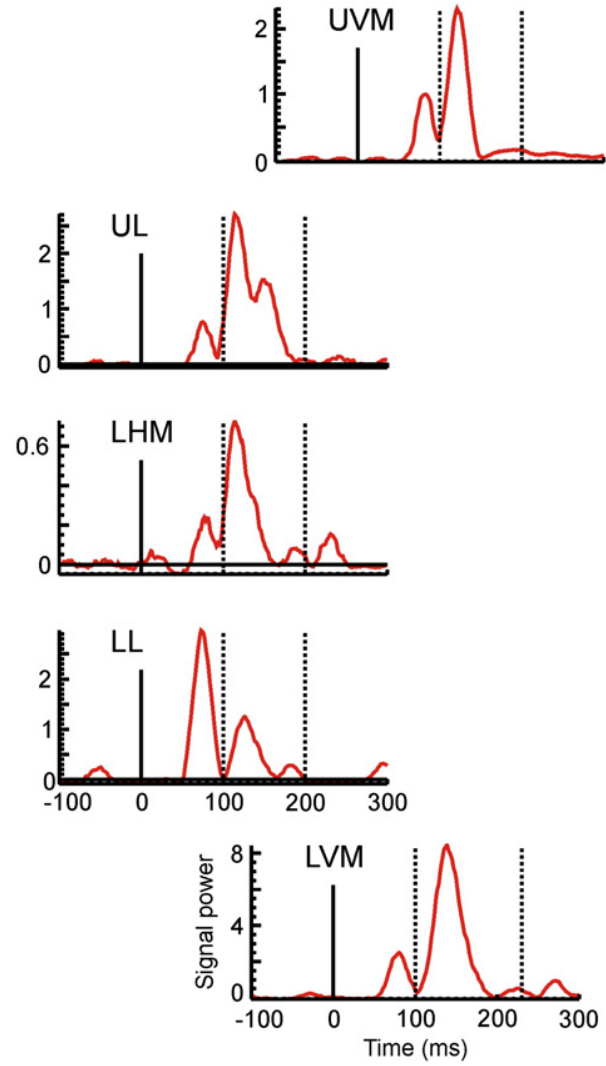

Fig. 5. SP time courses for V1 ROIs in the (right) intact hemisphere of GY. Each figure displays time course in the same format as in Fig. 4, but only for the visual fields: UVM, LVM, LL, LHM and UL. As in Fig. 4, each figure shows the time course for the ROI corresponding to the stimulated location.

in these three ipsilesional ROIs approximate those of the second-phase responses in the normal subjects. The responses from the damaged hemisphere of GY therefore suggest that in the absence of V1 the entire first visual processing phase is missing. The responses in each of these three high-level visual areas in the patient peaked roughly at the same latency (Fig. 9), similar to the first-phase responses in normal subjects (Fig. 10). The latencies of responses elicited by stimuli in the cortically blind contralateral hemifield were 10-20 ms longer compared to responses in the same areas elicited by stimuli in the sighted ipsilateral hemifield.

\section{Discussion}

Our results showed that the visual processing in normal subjects in the first $200 \mathrm{~ms}$ of the post-stimulus period proceeded in two phases, spanning roughly the first and second 100 -ms post-stimulus intervals, respectively, in good agreement with other studies (Ioannides and Poghosyan, 2012; Liu et al., 2002; Meeren et al., 2008; Sugase et al., 1999). In each phase the stimulus-evoked activity started in V1 and quickly spread through the dorsal and ventral streams toward higher levels of visual cortical hierarchy up to post-retinotopic extrastriate areas (Ioannides and Poghosyan, 2012).

The early visual processing elicited by normally perceived stimuli projected in the left hemifield of GY produced activity in his intact (right) occipital lobe which followed the spatiotemporal pattern observed in healthy subjects, including the canonical retinotopic organization (Baseler et al., 1999), response timing and sequence, and segmentation into two distinct processing phases. However, visual processing in the damaged (left) hemisphere for unseen right hemifield stimuli was qualitatively different. In fact, no cortical response was evident at latencies covering 

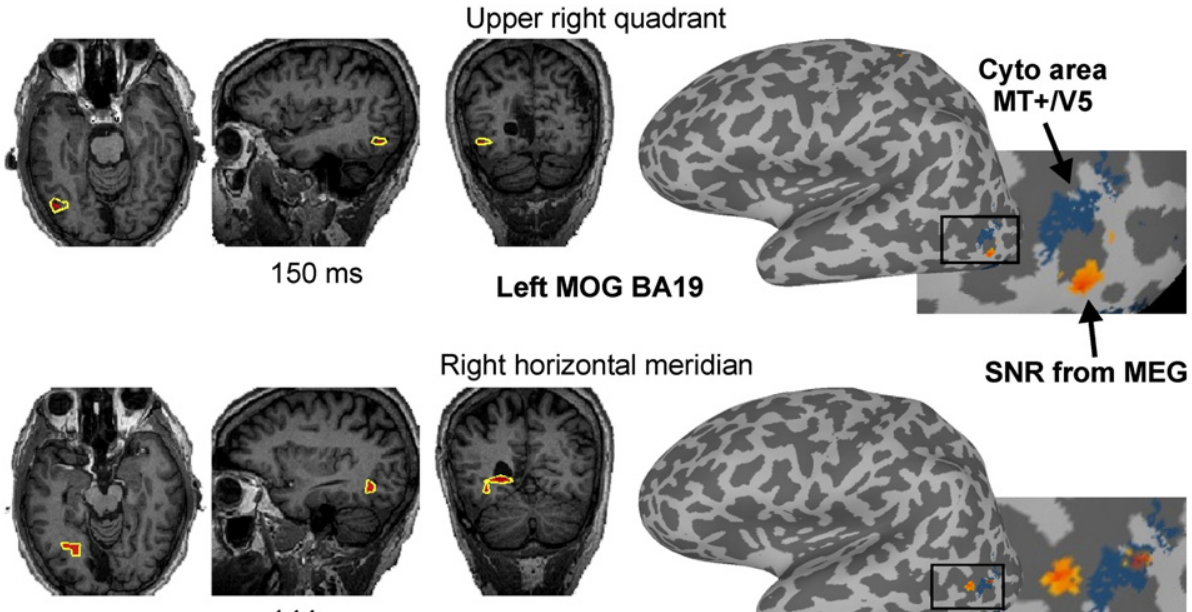

Right horizontal meridian

SNR from MEG
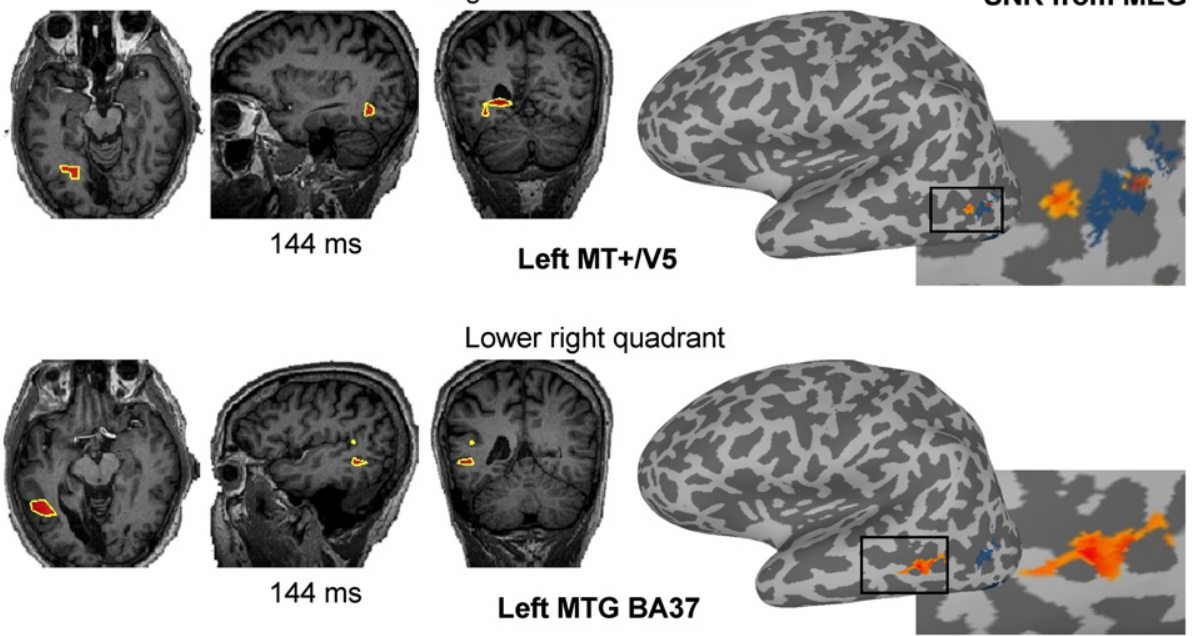

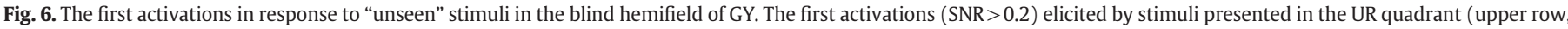

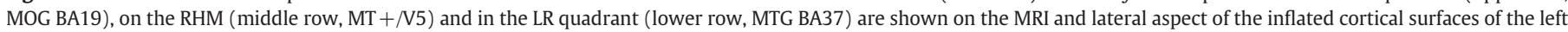

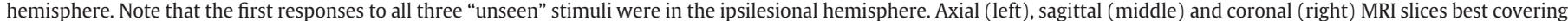

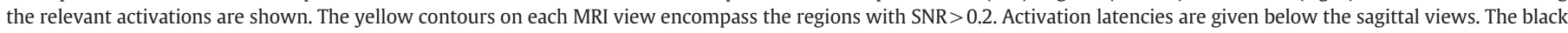

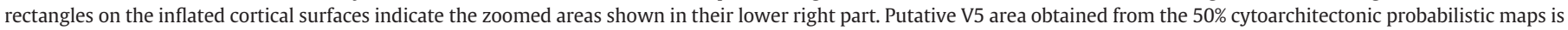
also shown in the inflated maps and they are captured also on the first and second zoomed views.

the first processing phase ( $<130 \mathrm{~ms})$. This clearly indicates that the responses in extrastriate visual areas observed in the intact brain during this phase are determined by the feedforward progression of activity initiated in V1. The first activations elicited by stimuli in the cortically blind hemifield occurred after $140 \mathrm{~ms}$ in the contralateral (ipsilesional) high-level extrastriate areas around MOG, MTG and STS. Following this first response, the activity spread forward rapidly through temporal and occipital cortices toward higher level areas and backward toward early retinotopic visual areas. The back-propagated activity reached earlier visual areas such as V2/3 in 10-20 ms. However, in contrast to responses in the intact hemisphere, this activity did not follow the classic retinotopy. Specifically, stimuli presented in the lower right quadrant of visual field (LR) elicited activity predominantly in the ventral V2/3, while the activity in response to stimuli presented in the upper right quadrant of visual field (UR) was in dorsal V2/3. This spatial organization of responses does not agree with the predictions of classic retinotopic model, according to which LR and UR stimuli should produce responses in dorsal and ventral portions of the early visual cortex respectively. However, the back-propagated activity may follow non-classic retinotopy resulted from the reorganization of the visual cortex following the long-term lesion of V1 (Rosa et al., 2000).

The delineation of early visual cortical areas of normal subjects provided here is consistent with the maps obtained routinely with fMRI. For each control subject, the V1-V2 borders (representations of the vertical meridian), estimated in the current study on the basis of source analysis of MEG data alone, were in excellent agreement with those obtained from an independent fMRI experiment. The correct estimation of the vertical and horizontal meridians, delineating the early visual areas, allowed assignment of responses and ROIs within cytoarchitectonic areas purely on the basis of the tomographic source analysis of MEG signals at least for V1, V2 and V3. The resulting spatial distribution and temporal sequence of evoked responses are in line with a feedforward sweep of activity through the visual hierarchy (Lamme and Roelfsema, 2000).

\section{Earlier studies}

Patient GY has been examined in numerous previous studies. The results we reported here are largely consistent with these previous findings, although a detailed comparison has not been always possible, either because different stimuli and protocols have been used or because previous techniques did not provide results with comparable timing and spatial accuracy. In the present study we have opted for stimuli known to produce a strong response in V1 so as to minimize the chances of missing weak activations initiated in "islands of preserved cortex" in early retinotopic areas that have been suggested as possible explanation of blindsight in several patients. Our results found no such evidence, thus strengthening the conclusions from earlier studies of GY (Kentridge et al., 1997; Weiskrantz, 2009a). The identification of activity in the damaged hemisphere in high-level areas is thus even more convincing because the stimuli we used do not usually give strong responses in these areas. The activation identified close to the MT+/V5 in our study is consistent with other studies using PET (Barbur et al., 1993), MEG (Holliday et al., 1997) and fMRI (Goebel et al., 2001) in the same patient. In the early PET (Barbur et al., 1993) and MEG (Holliday et al., 1997) studies the actual tasks and stimuli were rather different and no coordinates are available for detailed comparison. Based on the Talairach coordinates (Talairach and Tournoux, 1988) the MT+/V5 activity found in the fMRI study (Goebel et al., 2001) is $\sim 1.4 \mathrm{~cm}$ from our MT +/V5 ROI, and the activity 


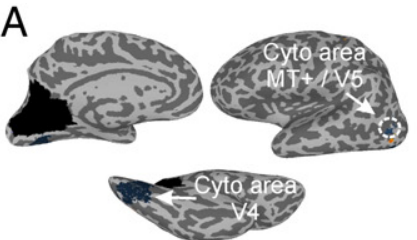

150

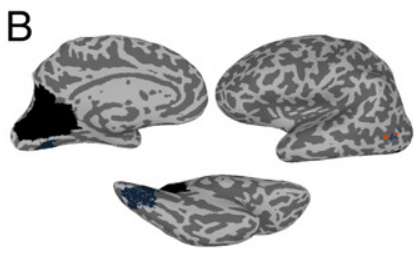

144

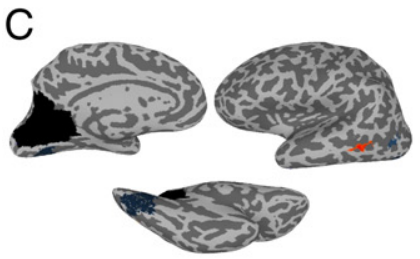

144

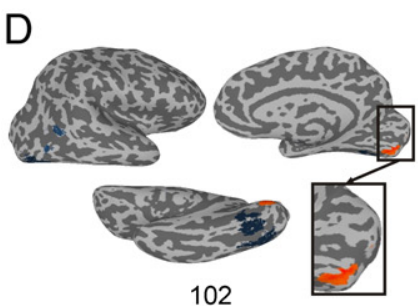

E

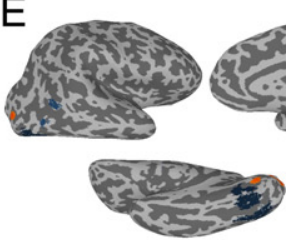

102

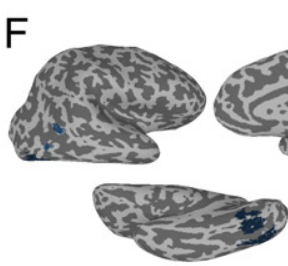

102

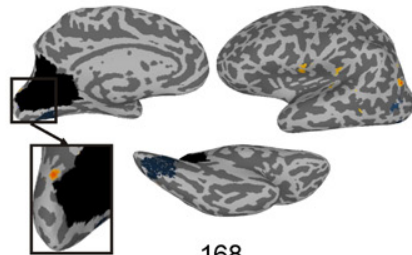

168

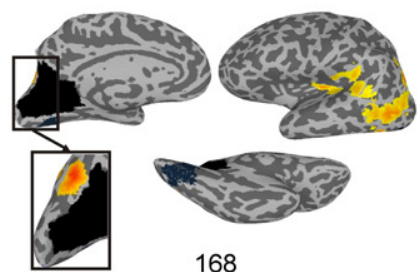

168

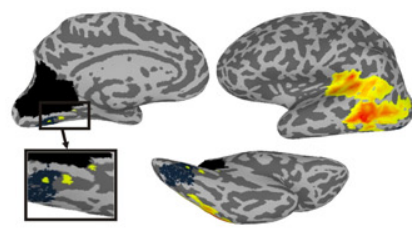

156
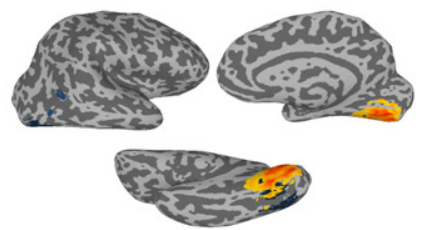

168
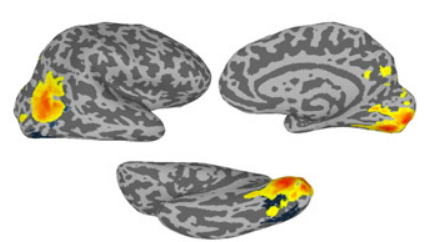

168
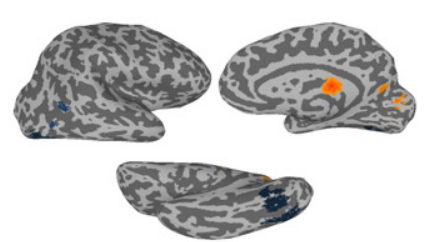

156
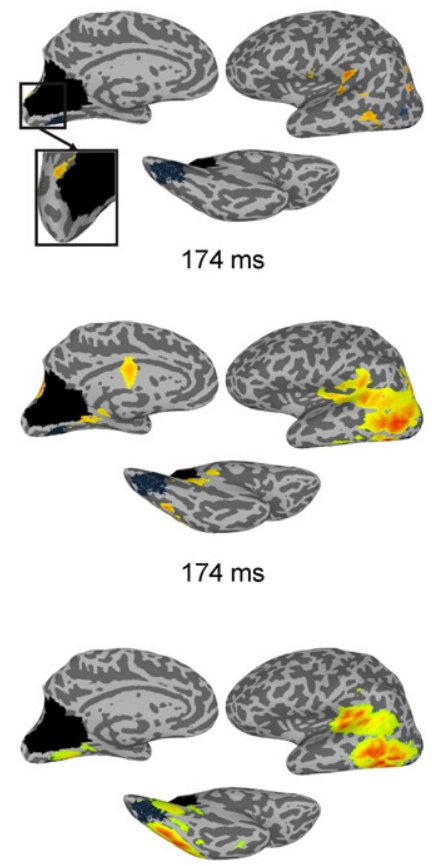

$162 \mathrm{~ms}$
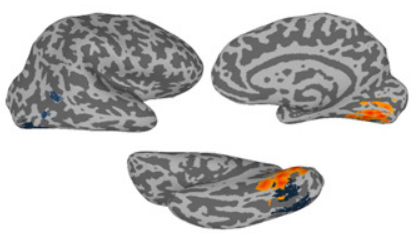

$174 \mathrm{~ms}$

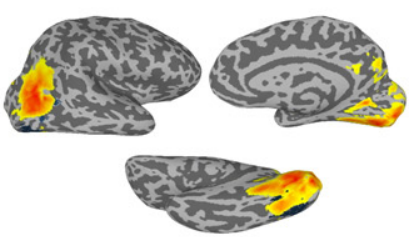

$174 \mathrm{~ms}$
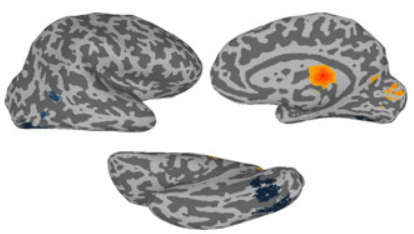

$162 \mathrm{~ms}$

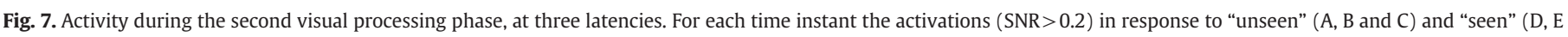

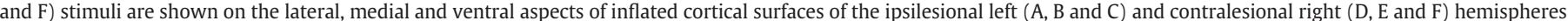

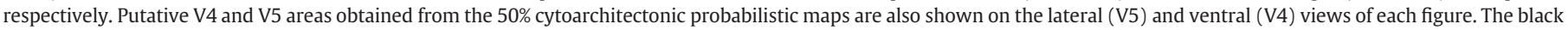

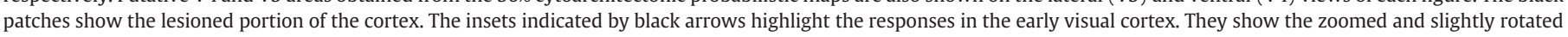

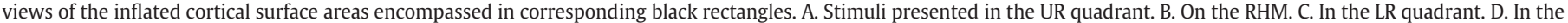
UL quadrant. E. On the LHM. F. In the LL quadrant.

Goebel et al. identified in response to coloured images of natural objects is $\sim 1.3 \mathrm{~cm}$ from our MOG ROI.

It is worth comparing our results with those of a combined fMRI and MEG study in another hemianopic patient with cortical blindness, admittedly of much younger age (Schoenfeld et al., 2002). The activity center for V5 from this study was $\sim 2.2 \mathrm{~cm}$ away from ours and the one for LOT $\sim 0.9 \mathrm{~cm}$ from our MOG activity. The timing of the peak activity elicited by stimuli in the blind hemifield was not quoted, but the beginning of the activity was similar to the one observed in our study, as was the observation that “... evoked activity occurred earlier in the higher-tier visual areas V4/V8 and V5 than in the lower-tier areas V2/V3 adjacent to the lesion."

Although the experiments discussed above differ in important ways to the one we did here, the overall consistency of the results across studies is 

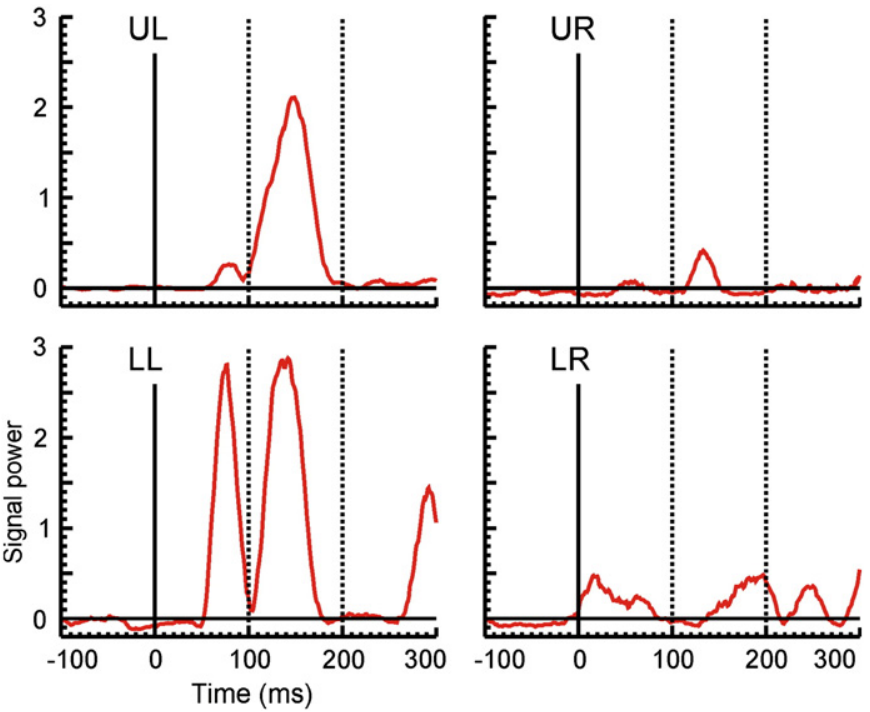

Fig. 8. SP time courses for estimated V2 ROIs in GY. Each figure displays time course for one quadrant of visual field (printed on its upper left part) for the corresponding ROI.

important, because it suggests that an access to higher level visual cortex, is available from the blind field and is not dependent on V1. Recent evidence from animal and human neuroimaging studies suggests the existence of at least two separate extra-geniculostriate pathways bypassing V1 and linked to functional and behavioral evidence of blindsight. One pathway involves direct connections between the intercalated layers of the lateral geniculate nucleus of the thalamus and area MT +/V5, which is especially involved in movement perception (Bridge et al., 2008; Schmid et al., 2010). The other pathway involves a disynaptic pathway
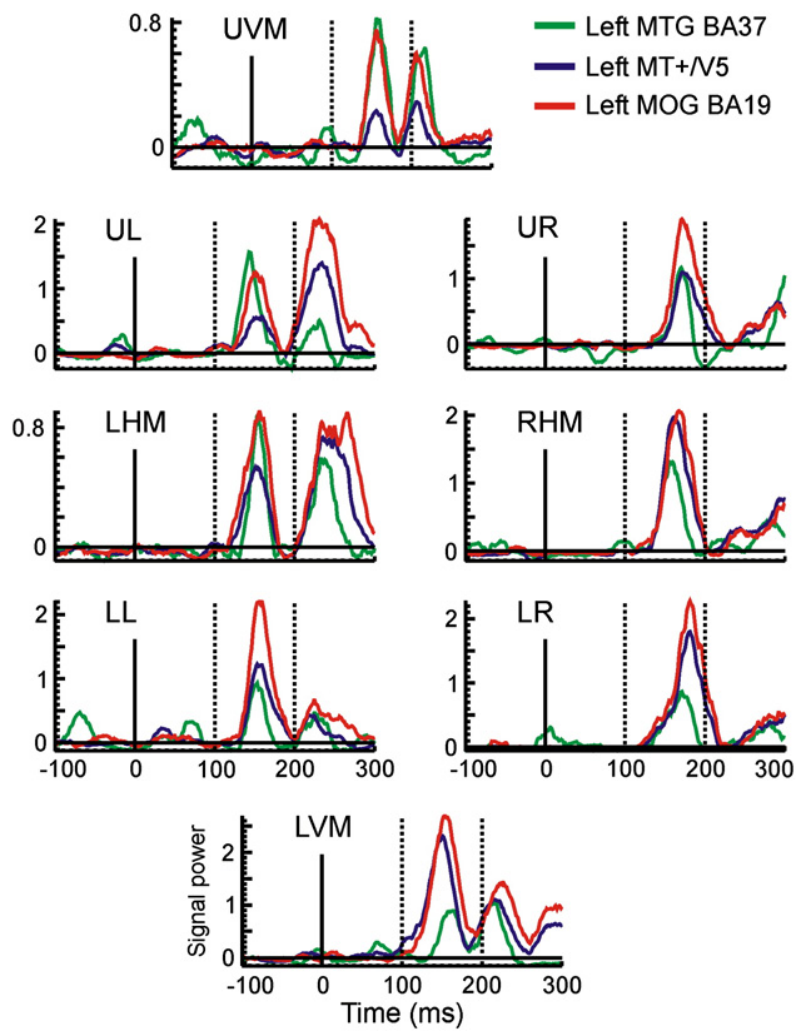

Fig. 9. SP time courses for the three post-retinotopic ipsilesional ROIs in GY. Time courses for the three ROIs are superimposed (red - left MOG BA19, blue - left $\mathrm{MT}+/ \mathrm{V} 5$, green - left MTG BA37). Each figure displays time courses for one location of the visual field, which is specified in its upper left part (UVM, UR, RHM, LR, LVM, LL, LHM and UL) and corresponds to its schematic location within the figure. connecting the superior colliculus to extrastriate occipito-temporal areas via the visual pulvinar, particularly active in visual-motor integration and "attention blindsight" (Lyon et al., 2010; Tamietto et al., 2010). The spatiotemporal precision of our results supports and extends these previous findings showing that, if this is indeed the way the stimulus effect reaches the blind hemisphere, this extra-geniculostriate access is activated during the second phase of visual processing and it spreads back into the earlier visual areas in a non-retinotopic manner and without precise time sequencing. We did not attempt to identify the subcortical origin of this extrastriate activity because the MEG hardware we used may not be very sensitive to deep sources. Another possible access to higher level visual cortex may be from neurons with very large receptive fields dispersed in early retinotopic cortex (e.g. in prostriata or V2 areas). These neurons may not form topographically well-defined groupings of neurons and hence produce no measurable MEG signal, but they may converge onto well-defined circumscribed regions in the high-level visual areas and thus produce the activations we have identified. The appearance of these activations in the second phase of processing may be due to a combination of the contribution from earlier visual areas and directly from subcortical routes, as both inputs might be needed to give rise to a measurable signal.

\section{Data analysis methodology}

In the present study we used tomographic source analysis (MFT) (Ioannides et al., 1990; Moradi et al., 2003; Papadelis et al., 2009; Poghosyan and Ioannides, 2007; Taylor et al., 1999) of single trial MEG signals to identify the precise spatiotemporal sequence of visual cortical responses following presentation of simple checkerboard patterns. MFT was used routinely to map activity in the brain of individual subjects for almost every MEG multichannel hardware, from the early systems covering only part of the head (Ribary et al., 1991) to most of the whole head systems used today, including the two used in the current study. Although MFT can reliably recover reasonably deep sources (Ioannides et al., 2005), in the current study we focused only on relatively superficial neural sources where the sensitivity of both MEG hardware is excellent. Separate experiments have demonstrated that activity within V1 can be reliably extracted and differentiated from activity in extrastriate areas when the V1-V2 border is available from an independent fMRI measurement (Moradi et al., 2003). More recently we also showed that information from cytoarchitectonic probability maps can be combined with the tomographic estimates (Papadelis et al., 2011), and that the spatiotemporal evolution of activity elicited by simple stimuli can be reliably and reproducibly extracted in different cytoarchitectonic areas (Poghosyan and Ioannides, 2007). Here, the analysis of simulated data has demonstrated that the activity in locations within V1 and V2 can be disentangled using the two different MEG systems. In this work we have also extended the methodology so as to define the borders of visual areas and map the activity within each visual area from the MEG data alone. We tested the new capability using fMRI V1-V2 borders for normal subjects and the cytoarchitectonic probabilistic maps for all subjects. While such a delineation of early visual cortical areas using MEG is a very valuable capability, more detailed tests and validation with fMRI-based retinotopic maps are necessary to confirm accuracy and reliability of the procedure. Taken together our results demonstrate the effectiveness of the methodology for both MEG systems used in this study and the results of the analysis of the real MEG data underscore their overall consistency with known properties of the visual system.

\section{Different MEG systems}

Could the use of different MEG hardwares in GY and normal subjects introduce spurious effects in our results? The rationale of our analysis is simple: our main comparison is between the intact and damaged hemispheres of GY. For this critical comparison, the issue of different MEG facilities does not apply. However, we first tested 

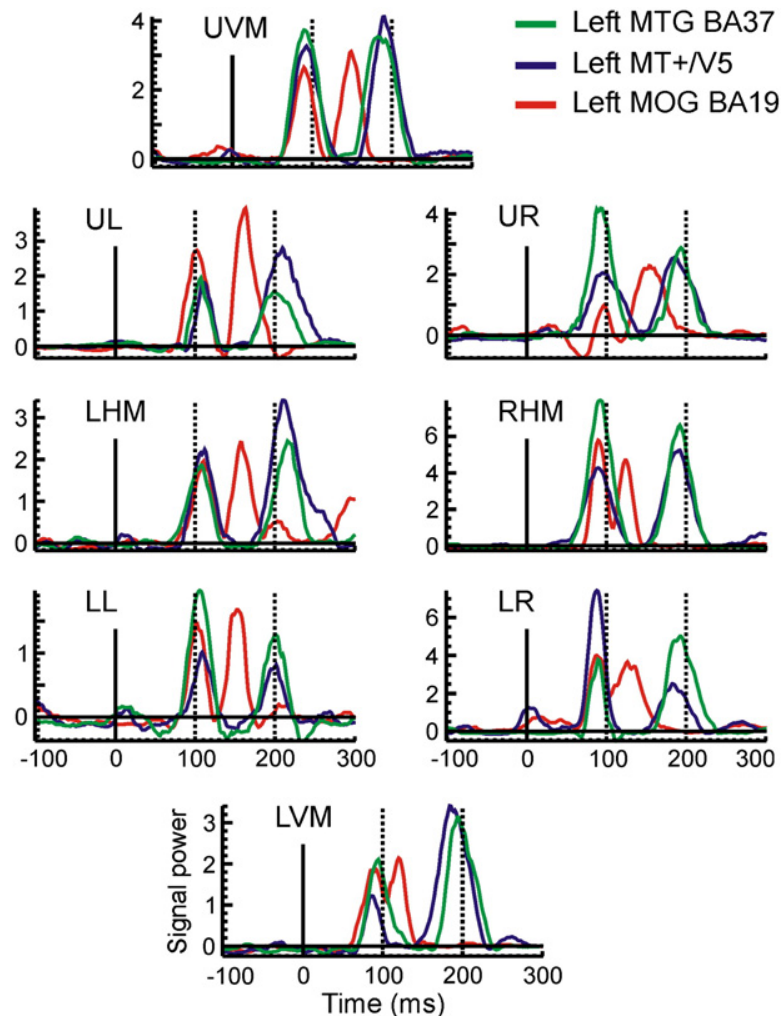

Fig. 10. SP time courses in a typical control subject for three ROIs corresponding to GY's three post-retinotopic ipsilesional ROIs shown in Fig. 8. Time courses for the three ROIs are superimposed (red - left MOG BA19, blue - left MT +/V5, green - left MTG BA37). Each figure displays time courses for one location of the visual field, which is specified in its upper left part (UVM, UR, RHM, LR, LVM, LL, LHM and UL) and corresponds to its schematic location within the figure. The results for stimuli presented at $9^{\circ}$ eccentricity are shown.

the hypothesis that the neural processing in the intact hemisphere of $\mathrm{GY}$ is consistent with what we have observed in our detailed study in normal subjects (albeit with another MEG hardware). Indeed, we found very similar spatiotemporal response patterns in the intact hemisphere of GY and in healthy subjects. If the result was different then one could justifiably argue that these differences were due to the differences in the hardware. The results were however similar and this, if anything, argues that there was no unexpected effect introduced by the use of different MEG systems on our results. The similarity of the activity patterns between the intact hemisphere of GY and healthy subjects, and the consistency with the previous literature shows that any possible spurious effect of different MEG hardwares is negligible. The key comparison between the intact and damaged hemisphere of GY is, of course, done with the same system.

At the time of the experiment with GY the MEG laboratory at RIKEN Brain Science Institute, where the experiments with normal subjects have been conducted, had been decommissioned. Therefore the experiment with GY was conducted in a different laboratory at ULB-Hôpital Erasme using identical parameters for the stimuli, except for small differences to ensure that stimuli presented in the right hemifield were entirely within the blind field of GY. All data were analyzed using a robust methodology (Data analysis methodology section) that was successfully applied before to data from different MEG systems, including studies with the two MEG systems used in the current study and studies on the same subjects with different MEG hardware (Ioannides et al., 2002). Part of the preparatory work for the current study included computer simulations (Computer simulations and Analysis of simulated data sections, and Supplementary material) and analysis of magnetic phantom measurements from the ULB-Hôpital Erasme MEG hardware (data not presented here).

\section{Other brain activations}

In addition to the identified ROIs that were robustly activated by our stimuli, two additional labile intermittent sources were evident in the intact hemisphere of GY: one source along the parieto-occipital sulcus (see Fig. $3 \mathrm{C}$ at $60 \mathrm{~ms}$ ) and another in 'deep' subcortical structures (see Fig. 3C at $66 \mathrm{~ms}$ ). An early, concurrent with V1, intermittent activity along the parieto-occipital sulcus has been reported also earlier (Portin et al., 1998; Tzelepi et al., 2001; Vanni et al., 2001). Studies have suggested this area to be the human homologue of monkey's V6 and reflect an automatic activation of a part of the attention- or visuomotor-related networks (Vanni et al., 2001).

The deep source on the other hand may be an artifact of the source localization process, or a reflection of a true subcortical source. The former explanation we consider unlikely, because the methodology we used here does not usually have such artifacts. It solves the inverse problem using five separate source spaces (see Tomographic source analysis section). The solutions are then combined together into a final source space that covers the entire brain. The use of regularization in solving the inverse problem reduces such 'ghost' images, but even in the case where the regularization is not enough to eliminate such sources, they will appear at different locations in each source space, and will be heavily penalized when the solutions from different source spaces are combined. Nevertheless, we cannot fully exclude such possibility.

The marked asymmetry between the brain responses to upper and lower visual field stimuli observed in the current study has been reported earlier in normal subjects (Fylan et al., 1997; Portin et al., 1999; Tzelepi et al., 2001) as well as in GY (de Gelder et al., 2001). Evidence suggests that upper and lower visual fields have different functional roles, with lower field superiority found in majority of tasks (Bilodeau and Faubert, 1997; Danckert and Goodale, 2001; Genzano et al., 2001; Rubin et al., 1996; Skrandies, 1987). However, upper field advantages in a number of tasks have also been reported (Goldstein and Babkoff, 2001; Zhou and King, 2002). Moreover, receptors and ganglion cells are denser in the human upper than lower hemiretina (Curcio and Allen, 1990; Curcio et al., 1990), ensuing an anatomical overrepresentation of the lower fields in the retina. This visual field specialization has been attributed to the adaptation of the human visual system to the environment in which we live (Previc, 1990; Skrandies, 1987).

\section{Conclusions}

Our results show that the response to "unseen" stimuli in a cortically blind patient with V1 lesion is delayed in time, does not adhere to classic retinotopic organization and appears first on the cortex in high-level extrastriate dorsal areas, whereas the spatiotemporal responses to the intact hemisphere are similar to the standard pattern observed in healthy subjects.

\section{Disclosure statement}

Authors declare no conflict of interest.

\section{Role of the funding source}

The funding agencies did not play any role in the study design, in the collection, analysis, and interpretation of data, in the writing of the report or in the decision to submit the paper for publication.

Supplementary data to this article can be found online at http:// dx.doi.org/10.1016/j.neuroimage.2012.07.058.

\section{Acknowledgment}

We thank patient GY for participating in the study. The experiments with the three control subjects were performed at the Laboratory for 
Human Brain Dynamics (1998-2009), RIKEN Brain Science Institute. This work was supported by the project "TANGO - Emotional interaction grounded in realistic context" under the Future and Emerging Technologies (FET) program from the European Commission (FP7-ICT-249858) to Beatrice de Gelder. It was co-funded by Cyprus Research Promotion Foundation grants ANOP $\Omega \Pi I \Sigma T I K E \Sigma / K O I N \Omega / 0308 / 16$, EПIXEIPH $\Sigma E I \Sigma /$ $\Pi$ ПОION/0609/76 and the European Regional Development Fund of the E.U. Marco Tamietto was supported by a Post-Doc Veni grant (451-07-032) from NWO (Netherlands Organization for Scientific Research) and partly from the Fondazione Carlo Molo, Torino, Italy. Xavier De Tiège is "Clinicien Chercheur Spécialiste" at the FRS-FNRS (Belgium).

\section{References}

Barbur, J.L., Watson, J.D., Frackowiak, R.S., Zeki, S., 1993. Conscious visual perception without V1. Brain 116, 1293-1302.

Baseler, H.A., Morland, A.B., Wandell, B.A., 1999. Topographic organization of human visual areas in the absence of input from primary cortex. J. Neurosci. 19, 2619-2627.

Bilodeau, L., Faubert, J., 1997. Isoluminance and chromatic motion perception throughout the visual field. Vision Res. 37, 2073-2081.

Bridge, H., Thomas, O., Jbabdi, S., Cowey, A., 2008. Changes in connectivity after visual cortical brain damage underlie altered visual function. Brain 131, 1433-1444.

Cheng, K., Waggoner, R.A., Tanaka, K., 2001. Human ocular dominance columns as revealed by high-field functional magnetic resonance imaging. Neuron 32, 359-374.

Cowey, A., Stoerig, P., 2004. Stimulus cueing in blindsight. Prog. Brain Res. 144, 261-277.

Curcio, C.A., Allen, K.A., 1990. Topography of ganglion cells in human retina. J. Comp. Neurol. 300, 5-25.

Curcio, C.A., Sloan, K.R., Kalina, R.E., Hendrickson, A.E., 1990. Human photoreceptor topography. J. Comp. Neurol. 292, 497-523.

Danckert, J., Goodale, M.A., 2001. Superior performance for visually guided pointing in the lower visual field. Exp. Brain Res. 137, 303-308.

de Gelder, B., Vroomen, J., Pourtois, G., Weiskrantz, L., 1999. Non-conscious recognition of affect in the absence of striate cortex. Neuroreport 10, 3759-3763.

de Gelder, B., Pourtois, G., van Raamsdonk, M., Vroomen, J., Weiskrantz, L., 2001. Unseen stimuli modulate conscious visual experience: evidence from interhemispheric summation. Neuroreport 12, 385.

De Tiège, X., Op de Beeck, M., Funke, M., Legros, B., Parkkonen, L., Goldman, S., Van Bogaert, P., 2008. Recording epileptic activity with MEG in a light-weight magnetic shield. Epilepsy Res. 82, 227-231.

Eickhoff, S.B., Stephan, K.E., Mohlberg, H., Grefkes, C., Fink, G.R., Amunts, K., Zilles, K. 2005. A new SPM toolbox for combining probabilistic cytoarchitectonic maps and functional imaging data. Neuroimage 25, 1325-1335.

Fisher, N.I., 1993. Statistical analysis of circular data. Cambridge University Press, Cambridge, MA.

Fylan, F., Holliday, I.E., Singh, K.D., Anderson, S.J., Harding, G.F., 1997. Magnetoencephalographic investigation of human cortical area V1 using color stimuli. Neuroimage 6, 47-57.

Genzano, V.R., Di Nocera, F., Ferlazzo, F., 2001. Upper/lower visual field asymmetry on a spatial relocation memory task. Neuroreport 12, 1227-1230.

Goebel, R., Muckli, L., Zanella, F.E., Singer, W., Stoerig, P., 2001. Sustained extrastriate cortical activation without visual awareness revealed by fMRI studies of hemianopic patients. Vision Res. 41, 1459-1474.

Goldstein, A., Babkoff, H., 2001. A comparison of upper vs. lower and right vs. left visual fields using lexical decision. Q. J. Exp. Psychol. A 54, 1239-1259.

Grill-Spector, K., Malach, R., 2004. The human visual cortex. Annu. Rev. Neurosci. 27, 649-677.

Hironaga, N., Ioannides, A.A., 2002. Accurate co-registration for MEG reconstructions. In: Nowak, H., Haueisen, J., Giessler, F., Huonker, R. (Eds.), Proceedings of the 13th International Conference on Biomagnetism. VDE Verlag, Berlin, pp. 931-933.

Holliday, I.E., Anderson, S.J., Harding, G.F., 1997. Magnetoencephalographic evidence for nongeniculostriate visual input to human cortical area V5. Neuropsychologia 35, 1139-1146.

Holmes, G., 1945. The representation of the visual field in human striate cortex. Proc. R. Soc. Lond. B 132, 348-361.

Horton, J.C., Hoyt, W.F., 1991. The representation of the visual field in human striate cortex. A revision of the classic Holmes map. Arch. Ophthalmol. 109, 816-824.

Ioannides, A.A., Poghosyan, V., 2012. Spatiotemporal dynamics of early spatial and category-specific attentional modulations. Neuroimage 60, 1638-1651.

Ioannides, A.A., Bolton, J.P.R., Clarke, C.J.S., 1990. Continuous probabilistic solutions to the biomagnetic inverse problem. Inv. Probl. 6, 523-542.

Ioannides, A.A., Liu, L.C., Khurshudyan, A., Bodley, R., Poghosyan, V., Shibata, T., Dammers, J., Jamous, A., 2002. Brain activation sequences following electrical limb stimulation of normal and paraplegic subjects. Neuroimage 16, 115-129.

Ioannides, A.A., Fenwick, P.B.C., Liu, L.C., 2005. Widely distributed magnetoencephalography spikes related to the planning and execution of human saccades. J. Neurosci. 25, 7950-7967.

Jahn, O., Cichocki, A., Ioannides, A.A., Amari, S., 1999. Identification and elimination of artifacts from MEG signals using extended independent component analysis. In: Yoshimoto, T., Kotani, M., Kuriki, S., Karibe, H., Nakasato, N. (Eds.), Recent Advances in Biomagnetism. Tohoku University Press, Sendai, pp. 224-228.

Kentridge, R.W., Heywood, C.A., Weiskrantz, L., 1997. Residual vision in multiple retinal locations within a scotoma: implications for blindsight. J. Cogn. Neurosci. 9, 191-202.
Lamme, V.A., Roelfsema, P.R., 2000. The distinct modes of vision offered by feedforward and recurrent processing. Trends Neurosci. 23, 571-579.

Laskaris, N.A., Ioannides, A.A., 2001. Exploratory data analysis of evoked response single trials based on minimal spanning tree. Clin. Neurophysiol. 112, 698-712.

Liu, J., Harris, A., Kanwisher, N., 2002. Stages of processing in face perception: an MEC study. Nat. Neurosci. 5, 910-916.

Liu, H., Agam, Y., Madsen, J.R., Kreiman, G., 2009. Timing, timing, timing: fast decoding of object information from intracranial field potentials in human visual cortex. Neuron 62, 281-290.

Lyon, D.C., Nassi, J.J., Callaway, E.M., 2010. A disynaptic relay from superior colliculus to dorsal stream visual cortex in macaque monkey. Neuron 65, 270-279.

Malikovic, A., Amunts, K., Schleicher, A., Mohlberg, H., Eickhoff, S.B., Wilms, M., PalomeroGallagher, N., Armstrong, E., Zilles, K., 2007. Cytoarchitectonic analysis of the human extrastriate cortex in the region of V5/MT+: a probabilistic, stereotaxic map of area hOc5. Cereb. Cortex 17, 562-574.

Meeren, H.K., Hadjikhani, N., Ahlfors, S.P., Hamalainen, M.S., de Gelder, B., 2008. Early category-specific cortical activation revealed by visual stimulus inversion. PLoS One 3 , e3503.

Moradi, F., Liu, L.C., Cheng, K., Waggoner, R.A., Tanaka, K., Ioannides, A.A., 2003. Consistent and precise localization of brain activity in human primary visual cortex by MEG and fMRI. Neuroimage 18, 595-609.

Okazaki, Y., Abrahamyan, A., Stevens, C.J., Ioannides, A.A., 2008. The timing of face selectivity and attentional modulation in visual processing. Neuroscience 152, 1130-1144.

Papadelis, C., Poghosyan, V., Fenwick, P.B.C., Ioannides, A.A., 2009. MEG's ability to localise accurately weak transient neural sources. Clin. Neurophysiol. 120, 1958-1970.

Papadelis, C., Eickhoff, S.B., Zilles, K., Ioannides, A.A., 2011. BA3b and BA1 activate in a serial fashion after median nerve stimulation: direct evidence from combining source analysis of evoked fields and cytoarchitectonic probabilistic maps. Neuroimage 54, 60-73.

Poghosyan, V., Ioannides, A.A., 2007. Precise mapping of early visual responses in space and time. Neuroimage 35, 759-770.

Poghosyan, V., Ioannides, A.A., 2008. Attention modulates earliest responses in the primary auditory and visual cortices. Neuron $58,802-813$.

Poppel, E., Held, R., Frost, D., 1973. Leter: Residual visual function after brain wounds involving the central visual pathways in man. Nature 243, 295-296.

Portin, K., Salenius, S., Salmelin, R., Hari, R., 1998. Activation of the human occipital and parietal cortex by pattern and luminance stimuli: neuromagnetic measurements. Cereb. Cortex 8, 253-260.

Portin, K., Vanni, S., Virsu, V., Hari, R., 1999. Stronger occipital cortical activation to lower than upper visual field stimuli. Neuromagnetic recordings. Exp. Brain Res. 124, 287-294.

Previc, F.H., 1990. Functional specialization in the lower and upper visual-fields in humans - its ecological origins and neurophysiological implications. Behav. Brain Sci. 13, 519-541.

Raz, J., Turetsky, B., Fein, G., 1988. Confidence intervals for the signal-to-noise ratio when a signal embedded in noise is observed over repeated trials. IEEE Trans. Biomed. Eng. 35, 646-649.

Ribary, U., Ioannides, A.A., Singh, K.D., Hasson, R., Bolton, J.P.R., Lado, F., Mogilner, A Llinas, R., 1991. Magnetic field tomography of coherent thalamocortical 40-Hz oscillations in humans. Proc. Natl. Acad. Sci. U. S. A. 88, 11037-11041.

Rosa, M.G., Tweedale, R., Elston, G.N., 2000. Visual responses of neurons in the middle temporal area of new world monkeys after lesions of striate cortex. J. Neurosci. 20, 5552-5563.

Rovamo, J., Virsu, V., 1979. Estimation and application of the human cortical magnification factor. Exp. Brain Res. 37, 495-510.

Rubin, N., Nakayama, K., Shapley, R., 1996. Enhanced perception of illusory contours in the lower versus upper visual hemifields. Science 271, 651-653.

Sahraie, A., Weiskrantz, L., Barbur, J.L., Simmons, A., Williams, S.C., Brammer, M.J., 1997. Pattern of neuronal activity associated with conscious and unconscious processing of visual signals. Proc. Natl. Acad. Sci. U. S. A. 94, 9406-9411.

Schmid, M.C., Mrowka, S.W., Turchi, J., Saunders, R.C., Wilke, M., Peters, A.J., Ye, F.Q. Leopold, D.A., 2010. Blindsight depends on the lateral geniculate nucleus. Nature 466, 373-377.

Schoenfeld, M.A., Noesselt, T., Poggel, D., Tempelmann, C., Hopf, J.M., Woldorff, M.G. Heinze, H.J., Hillyard, S.A., 2002. Analysis of pathways mediating preserved vision after striate cortex lesions. Ann. Neurol. 52, 814-824.

Schurger, A., Cowey, A., Tallon-Baudry, C., 2006. Induced gamma-band oscillations correlate with awareness in hemianopic patient GY. Neuropsychologia 44, 1796-1803.

Skrandies, W., 1987. The upper and lower visual field of man: Electrophysiological and functional differences. In: Ottoson, D. (Ed.), Progress in Sensory Phisiology, vol. 8. Springer-Verlag, Berlin, pp. 1-93.

Sugase, Y., Yamane, S., Ueno, S., Kawano, K., 1999. Global and fine information coded by single neurons in the temporal visual cortex. Nature 400, 869-873.

Talairach, J., Tournoux, P., 1988. Co-planar Stereotaxic Atlas of the Human Brain: 3Dimensional Proportional System: an Approach to Cerebral Imaging. Thieme, New York.

Tamietto, M., de Gelder, B., 2010. Neural bases of the non-conscious perception of emotional signals. Nat. Rev. Neurosci. 11, 697-709.

Tamietto, M., Castelli, L., Vighetti, S., Perozzo, P., Geminiani, G., Weiskrantz, L., de Gelder, B, 2009. Unseen facial and bodily expressions trigger fast emotional reactions. Proc. Natl. Acad. Sci. U. S. A. 106, 17661-17666.

Tamietto, M., Cauda, F., Corazzini, LL, Savazzi, S., Marzi, CA. Goebel, R., Weiskrantz, L de Gelder, B., 2010. Collicular vision guides nonconscious behavior. J. Cogn. Neurosci. 22, $888-902$.

Taylor, J.G., Ioannides, A.A., Mueller-Gaertner, H.W., 1999. Mathematical analysis of lead field expansions. IEEE Trans. Med. Imaging 18, 151-163. 
Tzelepi, A., Ioannides, A.A., Poghosyan, V., 2001. Early (N70m) neuromagnetic signal topography and striate and extrastriate generators following pattern onset quadrant stimulation. Neuroimage 13, 702-718.

Vanni, S., Tanskanen, T., Seppa, M., Uutela, K., Hari, R., 2001. Coinciding early activation of the human primary visual cortex and anteromedial cuneus. Proc. Natl. Acad. Sci. U. S. A. $98,2776-2780$.

Weiskrantz, L., 2009a. Blindsight: a Case Study Spanning 35 years And New Developments. Oxford University Press, Oxford, UK.

Weiskrantz, L., 2009b. Is blindsight just degraded normal vision? Exp. Brain Res. 192, 413-416.
Weiskrantz, L., Warrington, E.K., Sanders, M.D., Marshall, J., 1974. Visual capacity in the hemianopic field following a restricted occipital ablation. Brain 97, 709-728.

Weiskrantz, L., Barbur, J.L., Sahraie, A., 1995. Parameters affecting conscious versus unconscious visual discrimination with damage to the visual cortex (V1). Proc. Natl. Acad. Sci. U. S. A. 92, 6122-6126.

Zeki, S., Ffytche, D.H., 1998. The Riddoch syndrome: insights into the neurobiology of conscious vision. Brain 121, 25-45.

Zhou, W., King, W.M., 2002. Attentional sensitivity and asymmetries of vertical saccade generation in monkey. Vision Res. 42, 771-779. 